\title{
Freely chosen and instructed actions are terminated by different neural mechanisms revealed by kinematics-informed EEG
}

\author{
Shivakumar Viswanathan $\dagger^{* 1,2}$, Bin A. Wang $\uparrow^{1,4}$, Rouhollah O. Abdollahi ${ }^{1}$, \\ Silvia Daun ${ }^{1,3}$, Christian Grefkes ${ }^{1,2}$, Gereon R. Fink ${ }^{1,2}$
}

${ }^{1}$ Cognitive Neuroscience, Institute of Neuroscience and Medicine (INM-3), Research Centre Jülich, 52425, Jülich, Germany.

${ }^{2}$ Department of Neurology, University Hospital Cologne, 50924, Cologne, Germany.

${ }^{3}$ Heisenberg Research Group of Computational Biology, Department of Animal Physiology, Institute of Zoology, University of Cologne, 50674, Cologne, Germany.

${ }^{4}$ Department of Neurology, Ruhr-University Bochum, 44789, Bochum, Germany.

$\dagger$ Equal contribution

*Correspondence to: s.viswanathan@,fz-juelich.de 


\begin{abstract}
Neurophysiological accounts of human volition are dominated by debates on the origin of voluntary choices but the neural consequences that follow such choices remain poorly understood. For instance, could one predict whether or not an action was chosen voluntarily based only on how that action is motorically executed? We investigated this possibility by integrating scalp electroencephalograms and index-finger accelerometer recordings acquired while people chose between pressing a left or right button either freely or as instructed by a visual cue. Even though freely selected and instructed actions were executed with equal vigor, the timing of the movement to release the button was comparatively delayed for freely selected actions. This chronometric difference was six-times larger for the $\beta$-oscillations over the sensorimotor cortex that characteristically accompany an action's termination. This surprising modulation of an action's termination by volition was traceable to volition-modulated differences in how the competing yet non-selected action was represented and regulated.
\end{abstract}

\title{
KEYWORDS
}

Volition, Cognitive control, Motor control, EEG, Inter-hemispheric coupling, Beta-band rebound 


\section{Introduction}

A person's ability to autonomously and endogenously choose between alternative courses of action in daily life is a key presumption of many social, political, legal and economic arrangements. The current understanding of how the brain implements this important cognitive capability for voluntary action is, however, complicated by volition's multiple connotations (Libet et al. 1983; Wegner 2002; Roskies 2010). Due to the dominant focus on resolving ambiguities surrounding the neural origin of a voluntary choice (Haggard 2008; Soon et al. 2008; Desmurget et al. 2009; Bode et al. 2014; Schurger and Uithol 2015), the neurophysiological consequences of choosing an action voluntarily remain poorly understood. The unknown scope of volition's consequences raises a basic question (Becchio et al. 2014): is it possible to reliably predict whether or not an action was voluntarily selected based only on how that action was motorically executed? Here, we sought an answer to this question by investigating the execution of index-finger actions that were either selected freely or selected to comply with external instructions.

An influential experimental approach to investigate volition's neural substrate, both in healthy and clinical populations, has been to modulate the volitional content of a choice using external instructions (Kutas and Donchin 1980; Libet, Wright, and Gleason 1982; Frith et al. 1991). This strategy's central rationale is that an action selected to comply with an instruction (such as a sign to "Turn right" at a crossroad) is less free than an action selected based on a whimsical subjective preference ("I want to turn right"). Based on this rationale, numerous studies have compared neural systems engaged by instructed and uninstructed (i.e., selfselected) actions using experimental paradigms that modulate how external instructions inform choices between simple discrete actions (e.g., pressing a button with either the left or right index 
finger) (Frith et al. 1991; Jahanshahi et al. 1995; Jenkins et al. 2000; Cunnington et al. 2002; Waszak et al. 2005; Van Eimeren et al. 2006; Mueller et al. 2007; Hoffstaedter et al. 2014; Ariani, Wurm, and Lingnau 2015; Michely et al. 2015; Wisniewski, Goschke, and Haynes 2016). In these comparisons, volition has been associated with brain structures, especially in the medial prefrontal cortex, that exhibit elevated activity when an action is self-selected relative to when that action is instructed (Krieghoff et al. 2011; Brass et al. 2013; Zapparoli, Seghezzi, and Paulesu 2017). However, these findings have been controversial and the topic of an extensive debate due to the difficulty in specifying and interpreting how actions were "self-selected" in these experiments and the numerous confounding differences (Lau et al. 2004; Botvinick, Cohen, and Carter 2004; Nachev et al. 2005; Nachev and Husain 2010; Passingham, Bengtsson, and Lau 2010; Schüür and Haggard 2011; Bode, Bogler, and Haynes 2013; Brass et al. 2013). An example of such a confounding difference is the sensory and perceptual demand to identify a stimulus-defined instruction that has no equivalent for freely selected actions. Furthermore, the decision about what action to choose and the timing of when to act are independent for a freely selected action unlike a stimulus-defined instruction that can ambiguously define both decisions (Schouten and Bekker 1967; Brass and Haggard 2008; Hoffstaedter et al. 2013) Nevertheless, despite these theoretical challenges, the experimental strategy of comparing instructed and uninstructed actions provided a pragmatic framework to assess volition's consequences, if any, for the motor execution of a selected action.

In a typical volition modulation paradigm, each trial requires a binary choice between pressing either a left or a right button with the index finger of the corresponding hand. The physical pressing of a button is presumed to follow the cognitive selection of one action over the other. Therefore, a successfully measured button-press event is a post-hoc behavioral indicator 
that a choice was in fact made on that trial. Besides, this event is also a marker of the choice's identity (left or right) and timing relative to a reference event, such as the previous action or the onset of the instruction stimulus. However, a button-press event is not the same as the index finger flexion/extension movements required to actually push the button down and then release it (Oulasvirta, Kim, and Lee 2018). A key assumption in prior studies has been that these indexfinger movements to press a button would be unaffected by whether the decision to press that button was self-selected or instructed. Indeed, a violation of this assumed brain/behavior relationship would pose the challenge of explaining the neural logic of how volition's role in expressing a preference between alternative actions (i.e., choose $A$-or- $B$ ?) could possibly influence the physical execution of the selected action (i.e., execute $A$-not- $B$ ) (Obhi and Haggard 2004; Becchio et al. 2014).

Here, we investigated volition's predicted absence of movement consequences using data from a previously conducted electroencephalography (EEG) study (Popovych et al. 2016; Wang et al. 2017)(see Section 2). In brief, EEG and index finger accelerations were concurrently recorded while participants made binary action choices in two contexts (Fig. 1A). In the Instructed context, a visual stimulus depicted either a left- or right-pointing arrow on each trial to indicate the required action. In the Free context, actions were self-selected without prompting stimuli. So, is it possible to distinguish between actions executed in the Free or Instructed context based on the brain/behavior dynamics that accompany index finger movements to push and then release a button? 

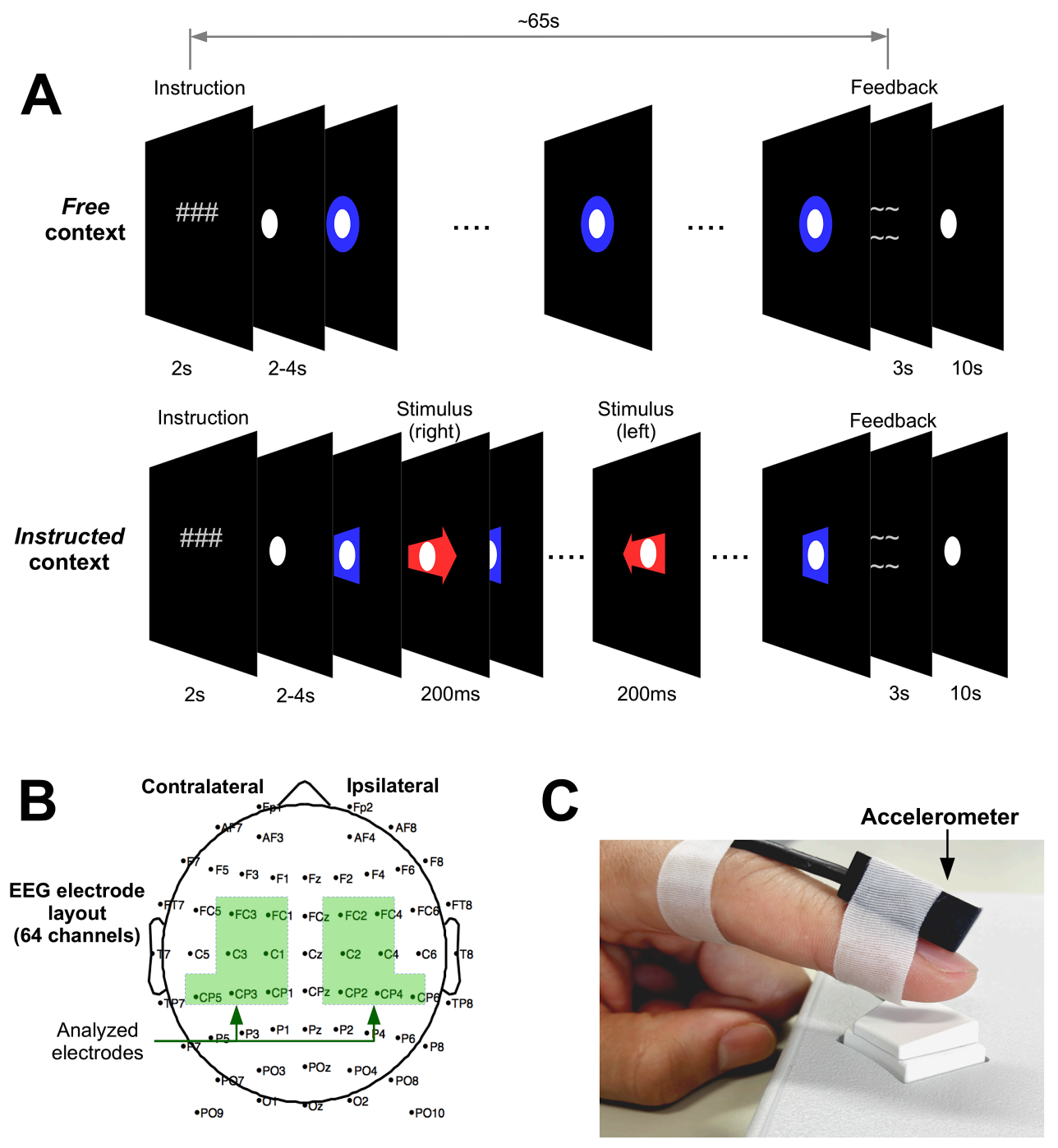

Figure 1. Experimental paradigm. (A) A schematic of the trial timing for one block of the Free and Instructed contexts. A white fixation dot was displayed on the screen at all times and was augmented with a concentric blue disc in the Free context and a blue square in the Instructed context to uniquely identify each context. (B) Green polygons indicate electrodes of interest. EEG from left and right hand trials were collapsed over corresponding electrodes contralateral to the moving finger (similarly for ipsilateral and medial electrodes), with electrodes over the left (right) hemisphere corresponding to contralateral (ipsilateral) electrodes respectively. (C) Accelerometers were firmly taped to the dorsal tip of each index finger. 


\section{Materials and methods}

\subsection{Participants}

Twenty-one healthy individuals (10 females, 11 males; age range: 22-35 years) participated in our experiment and received financial compensation. Participants had normal color vision, were right-handed (mean handedness score $=0.9$, according to the Edinburgh Handedness Inventory (Oldfield 1971)), and had no prior history of psychiatric or neurological disease. All participants provided written informed consent prior to the experiment. The local ethics committee of the Faculty of Medicine, University of Cologne, approved the study protocol. Data from 3 participants were excluded from the analysis based on poor quality of EEG data (see Section 2.6.3). All results reported here were based on data from the remaining 18 participants.

\subsection{Apparatus and data acquisition}

Stimuli were generated and displayed using the software Presentation ${ }^{\circledR}$ (version 11.0, Neurobehavioral Systems, Berkeley, CA) on a $47 \mathrm{~cm}$ x $29 \mathrm{~cm}$ LCD monitor with a screen resolution of $1280 \times 1024$ pixels, and a refresh rate of $60 \mathrm{~Hz}$. Index-finger button-presses were registered by two LUMITouch ${ }^{\mathrm{TM}}$ keypads (Photon Control Inc., Burnaby, BC, Canada), one for each hand.

Scalp EEG was recorded using a 64-channel active electrode system (actiCAP, Brain Products GmbH, Munich, Germany) mounted in a spherical array according to the extended 1020 international system (Fig. 1B). Three of the 64 electrodes (FT9, FT10 and TP10 in the 10-20 system) were not placed on the scalp but at the bilateral outer canthi and under the left eye to record bilateral horizontal and left vertical electro-oculograms (EOG). All electrodes were referenced online to the left mastoid. All electrode impedances were brought below $15 \mathrm{k} \Omega$ prior 
to the start of the experimental recording. The instantaneous acceleration of the left and right index finger was continuously recorded with an accelerometer (size: $22 \times 14 \times 8 \mathrm{~mm}^{3}$, weight: 8 gms) (Brain Products GmbH, Munich, Germany) attached to the dorsal-tip of each index finger (Fig. 1C). The $X, Y$ and $Z$ components of the measured acceleration (in a Cartesian coordinate system centered at the fingertip) were converted into voltage signals (sensitivity: $420 \mathrm{mV} / \mathrm{g}$, gain: 3.5) and integrated into the EEG recording as additional channels.

Voltages from the scalp-EEG and accelerometer channels were amplified, band-pass filtered from $0.1 \mathrm{~Hz}$ to $250 \mathrm{~Hz}$, and digitized at a sampling rate of $2500 \mathrm{~Hz}$.

\subsection{Paradigm}

The experiment had two movement related contexts (namely, the Free and Instructed contexts) and one non-movement control condition (namely, the Vision-only context). Although the current study focused exclusively on movement-related phenomena in the Free and Instructed context, details of the Vision-only context are reported here for completeness. Trials in each context were organized into blocks and structured as shown in Figure 1A. A white fixation dot was displayed on the screen at all times and was augmented with a concentric blue disc during the Free context and a blue square during the Instructed context as a reminder of the context currently underway.

On each trial of the Instructed context, the visual stimulus was a red arrow (width: $2^{\circ}$ visual angle, height: $1.2^{\circ}$ visual angle), which pointed either to the right or to the left and was displayed at the center of the screen for $200 \mathrm{~ms}$. Participants had to respond to this stimulus by pressing a button with the index finger corresponding to the arrow's direction, i.e., left or right. To ensure that these stimuli were indeed modulating action choices, task instructions 
emphasized a minimum required accuracy of $90 \%$. At the end of each block, a feedback screen displayed the numerical percentage accuracy value if the accuracy on that block was below $90 \%$ or the message - "Well done" - if the accuracy was greater than $90 \%$.

The Vision-only context was identical to the Instructed context in stimulus materials and display parameters, but participants were instructed to pay attention to the arrow stimuli without making a corresponding response.

In the Free context, there were no visual stimuli displayed apart from the continuously present fixation symbol. Participants were instructed to voluntarily produce single index finger button presses over the entire block.

As actions in the Free context were initiated without external stimuli, an important concern was that (i) the total number of responses, (ii) the proportion of right and left hand responses, and (iii) the interval between consecutive responses could vary in an idiosyncratic manner from block to block. This could in turn limit the comparability of the Free and Instructed contexts. Therefore, to increase inter-context comparability, participants were instructed to initiate a response at intervals of approximately 4 to 8 seconds, and to approximately balance the number of left and right index finger button presses over the block, while avoiding stereotyped response patterns and mental counting of the responses or the timeintervals. The feedback screen at the end of each block provided information on performance relative to the desired response timing and balance. The feedback messages were "Timing: Well done" when the total number of responses on that block $N=10 \pm 2$ responses (based on the ideal rate of a response every 6 s over the 60s block), "Timing: Too fast" for $N>12$ responses, or "Timing: Too slow" for $N<8$ responses. The extent of unbalanced responses in a block was estimated as, $100 \mathrm{x}\left|N_{\text {right }}-N_{\text {left }}\right| /\left(N_{\text {right }}+N_{\text {left }}\right)$, where $N_{\text {right }}$ and $N_{\text {left }}$ were the number of right and 
left index finger button presses, respectively. The corresponding feedback messages were "Balance: Well done" when this imbalance value was $\leq 30 \%$ (chosen as a soft threshold to avoid overly constraining participants behavior), or "Balance: Unbalanced" for imbalance values greater than $30 \%$.

\subsection{Adaptive trial design}

The total task period of the experiment (approx. $70 \mathrm{~min}$ ) consisted of 48 blocks of trials divided into 4 runs, each having 12 blocks ( 3 contexts $\mathrm{x} 4$ blocks). Unknown to the participants, an online adaptive procedure matched the number of left and right responses and the inter-response intervals in the Free and Instructed contexts to increase inter-context comparability (Michely et al. 2015, 2012). Blocks in each run were organized into triplets where a Free context block always occurred first, followed by the Instructed and Vision-only contexts, in randomized order. In each block of the Free context, the number of responses produced with each hand (i.e., $N_{\text {right }}$ and $\left.N_{\text {left }}\right)$ and the inter-response intervals were recorded. The recorded quantities $N_{\text {right }}$ and $N_{\text {left }}$ were then used to define the number of right-pointing and left-pointing arrows in the Vision-only and Instructed blocks that followed. The order of the recorded inter-response-intervals was randomized and these intervals were then used as the inter-stimulus intervals in the Instructed and Vision-only contexts. Intervals shorter than 4 s were replaced with randomly chosen intervals in the range 4 to $8 \mathrm{~s}$. 


\subsection{Procedure}

The EEG cap was first secured on the participant's head. Next, during the application of electrode gel to individual electrodes of the EEG cap, participants received verbal instructions and training on the experimental tasks.

All participants were trained in the same sequence of stages. First, the experimenter used a simulation of the actual experiment to familiarize participants with the visual information that they would encounter over the entire experiment (for example, the types of instruction and feedback screens, types of stimuli and fixation points). In the second stage, participants trained at responding to stimulus instructions (namely, left and right-pointing arrows) as required by the Instructed context. These stimuli were presented at intervals in the range 4-8s, which was the desired inter-response period for the Free context. In the third stage, the objective was to provide participants with a concrete sense for a 4-8s period. Participants were informed that stimuli would be displayed at intervals exactly as in the previous stage but, rather than respond to these stimuli, their task was to predict when each stimulus would be displayed and press a button to coincide with the stimulus onset. Training on the Free context followed in the fourth stage. Participants were instructed to freely press a button of their choice, with the guideline to act at intervals that were approximately similar to the "prediction" task that they had just been trained on. Training lasted until participants achieved a suitable response rate and were deemed to have a clear understanding of the instructions. Finally, participants were again shown the unique fixation symbols that they would encounter and had to identify the corresponding context for each fixation symbol correctly.

Following training, participants were instructed to minimize eye-blinks, to maintain fixation at all times during the task blocks, and to minimize unnecessary movements of their 
index fingers, head and body. The accelerometer was originally intended to precisely record the onset of index finger movements. Therefore, instructions emphasized the need for brief, decisive movements irrespective of context and the avoidance of anticipatory and unnecessary finger movements.

The experimental recordings were conducted in a darkened soundproof room. Participants were seated in a comfortable chair with their head supported by a chin-rest. Accelerometers were firmly attached to the dorsal tip of both index fingers with adhesive tape. The keypads were placed in a recess under the table where participants placed their hands so that the buttons were pressed without visibility of the index-finger movements. During the experiment, the experimenter continuously monitored the participants via a video camera to ensure that they maintained fixation, minimized eye-blinks, and stayed awake. After the experiment, participants were debriefed to verify their compliance with the instructions.

\subsection{EEG and accelerometer data preprocessing}

EEG and accelerometer data were preprocessed and analyzed using the EEGLab toolbox (Delorme and Makeig 2004) and custom scripts written in MATLAB (version MatlabR2015b, MathWorks Inc., Natick, Massachusetts, USA).

\subsubsection{Preliminary inspection}

Raw data were first bandpass filtered from 0.5 to $48 \mathrm{~Hz}$ (to remove low frequency trends and $50 \mathrm{~Hz}$ line noise) and then downsampled to $200 \mathrm{~Hz}$. Next, the continuous EEG data were visually inspected to exclude time-periods contaminated by paroxysmal and muscular artifacts unrelated to eye blinks. The accelerometer recordings were also inspected to exclude trials 
containing extraneous finger movements prior to the button-press, as well as trials with mirror movements.

\subsubsection{Movement onset identification}

The movement onset on each trial was identified from the continuous accelerometer recordings. The first derivatives of the acceleration along the $X, Y$, and $Z$ axes were computed and then combined to obtain the scalar (Euclidean) magnitude of the instantaneous acceleration change at each time point. This time-series was then smoothed and rescaled. Movement onset was defined using a threshold that was set to identify the earliest time point in a $125 \mathrm{~ms}$ window prior to each button press that showed a continuous increase in acceleration rate. All trials where the movement onset could not be unambiguously detected within this $125 \mathrm{~ms}$ pre-button window were excluded from further analyses. The threshold value was determined by visual inspection and the same threshold was used for all trials and participants. The identified movement onsets were integrated into the continuous EEG and accelerometer data.

\subsubsection{Epoching and artifact rejection/correction}

The inspected continuous EEG and accelerometer data were segmented into epochs relative to the movement onset on each trial, estimated from the accelerometer recordings. The epoch length for each context was chosen so that the epochs were non-overlapping and were long enough to have a baseline period of $1 \mathrm{~s}$. In the Free context, due to the ambiguous onset of the readiness potential (or Bereitschaftspotential) before the movement onset (Kornhuber and Deecke 1964; Shibasaki and Hallett 2006), epochs were defined from $-2.5 \mathrm{~s}$ to $+1.5 \mathrm{~s}$ relative to the movement onset, with a baseline period from $-2.5 \mathrm{~s}$ to $-1.5 \mathrm{~s}$. As described in section 2.4 , the 
inter-response intervals in the Free context were used to define inter-stimulus intervals in the Instructed contexts. To accommodate this constraint, epoch lengths in the Instructed context were 500ms shorter than in the Free context to ensure that consecutive epochs did not overlap and that no stimuli were displayed during the baseline period. Epochs were defined from $-2.0 \mathrm{~s}$ to $+1.5 \mathrm{~s}$ relative to movement onset with a baseline period from $-2 \mathrm{~s}$ to $-1 \mathrm{~s}$. Since an action in the Instructed context could not be correctly selected before the stimulus display, we assumed that these shorter epochs would not affect comparability to the longer epochs of the Free context. To ensure a stimulus-free baseline period, all trials in the Instructed context with a response time greater than $1 \mathrm{~s}$ were excluded from the final analysis

The epochs obtained after segmentation were again examined for artifacts. Epochs having amplitudes larger than $100 \mu \mathrm{V}$ over the entire epoch or with abnormal drifts greater than $75 \mu \mathrm{V}$ were rejected. Next, a semi-automated procedure based on an independent component analysis (ICA) was used to identify epochs contaminated by blinks, eye movements, muscle activity, and infrequent single-channel noise. The independent components were identified using the Infomax ICA algorithm implemented in EEGLAB. The ADJUST algorithm (Mognon et al. 2011) was then used to identify and reject components containing blink/oculomotor-related and other artifacts distinguishable from the rest of brain activity. Noisy channels were detected automatically by EEGLAB and interpolated using spherical spline interpolation. Subsequently, the artifact-free trials were average-referenced and baseline-corrected relative to the first $1 \mathrm{~s}$ of the epoch.

At this stage, data from three participants were excluded from further analyses because of an insufficient number of trials satisfying the quality criteria described above. One participant's data had a large number of epochs contaminated by eye movement artifacts; and 
data from two participants had many epochs contaminated by extraneous movements during the baseline period as detected by the accelerometers. The data of each of the remaining 18 participants had at least 100 artifact-free trials per context (100-160 Free trials, 104-161 correct Instructed trials).

\subsubsection{Spatial filtering}

The final EEG preprocessing stage was spatially filtering of the epoched EEG data using the surface Laplacian procedure. This procedure helps exclude low spatial frequency signals thus reducing the effects of distal generators due to volume conduction and increasing local spatial resolution at the electrodes (Perrin et al. 1989; Nunez et al. 1994; Cohen 2014). In our study the surface Laplacian was used specifically to (i) enhance topographic localization over the electrodes of interest (clustered bilaterally over the sensorimotor cortex as shown in Fig. 1B) and (ii) limit the influence of volume conducted signals at these electrodes, especially from occipital/parieto-occipital sources involved in sensory processing in the Instructed context. Surface Laplacians were estimated from the individual artifact-free EEG epochs using a spherical spline algorithm (Perrin et al. 1989) as implemented by the Current Source Density (CSD) toolbox (Kayser and Tenke 2006). CSD waveforms were computed for each original surface EEG potential (50 iterations, spline degree $=4$ ).

\subsubsection{Push/Release identification}

The epoched accelerometer data were used to identify the Push and Release movements on each trial. Pressing the button required a rapid flexion of the index finger to push the button down and, after a brief delay, an extension to release the button. The absolute acceleration of these 
Push and Release movements had distinctive peaks that could be identified from the recorded accelerometer data on each trial.

For each epoch, the scalar magnitude of the (Euclidean) acceleration at each time point was first computed from component accelerations along the $X, Y$ and $Z$ axes. The identification of Push and Release acceleration peaks was restricted to the time range $[0 \mathrm{~ms},+1000 \mathrm{~ms}]$ following movement onset. The mean acceleration over the $[-500 \mathrm{~ms}, 0 \mathrm{~ms}]$ period was subtracted from the absolute acceleration time-series to correct for small inter-trial baseline differences. To account for individual differences, the movement identification procedure had two stages that were applied to each participant's movements in each context. The objective of the first stage was to estimate a characteristic time point dividing the Push and Release movements across all trials for each context and participant. In the second stage, the estimated dividing time from the first stage was applied to each trial of that context.

The first estimation stage was implemented a follows. As a starting approximation, the Push and Release acceleration peaks were assumed to occur at least 40ms apart from each other. Since the maximum acceleration for the Push movement was typically higher than for the Release movement, the $40 \mathrm{~ms}$ constraint was necessary to distinguish the representative peak for the Release movement from the multiple peaks associated with the Push movement due to high frequency acceleration changes. For each participant and context, we calculated a maxAcc timeseries consisting of the maximum acceleration at each time-point across all epochs. This timeseries was used to estimate the time-points of two acceleration maxima: (1) the time point of the maximum acceleration value over the entire $\operatorname{maxAcc}$ time-series (2) the time point of the next highest acceleration value that was at least $40 \mathrm{~ms}$ distant from the first time-point. These two maxima were reference time points for the Push and Release movements. The characteristic 
dividing time-point between the Push and Release movements was defined as the time point of the minimum acceleration between the above two maxima. In the second stage of our procedure, the estimated dividing time from the first stage was applied to each trial. For each trial, the time and magnitude of the maximum acceleration before and after the dividing time were treated as the peak acceleration of the Push and Release movements, respectively. We confirmed the successful operation of this identification procedure by visually inspecting the resulting Push and Release estimates for each trial and for each participant.

The Push and Release peak on each trial was described by the respective magnitude and latency relative to movement onset. To obtain a robust estimate of each participant's mean acceleration and latency of the Push and Release movement per response hand in each context, we used a trimmed mean with a $15 \%$ cutoff (i.e., excluding $7.5 \%$ of the lowest and highest values) (R Ratcliff 1993). Accelerometer data from left and right index-finger movements were highly similar and were collapsed together for all analyses.

\subsection{ERP time-locking}

The epoched time-series were averaged to obtain event-related potentials (ERPs). Since the epochs were defined relative to the movement onset, the resulting ERPs are referred to here as movement-related ERPs. The Push-evoked and Release-evoked ERPs were obtained by averaging the epoched time-series relative to the time on each trial's Push acceleration peak and the Release acceleration peak respectively. 


\subsection{Oscillations}

The instantaneous phase of $\delta$-band oscillations $(2-4 \mathrm{~Hz})$ and instantaneous amplitude of $\beta$-band oscillations $(13-30 \mathrm{~Hz})$ was computed as follows. The preprocessed data were band-pass filtered in the required frequency range using a two-pass procedure with a Hamming windowed sinc FIR filter (as implemented by EEGLab). The Hilbert transform (Freeman 2004; Cohen 2014) was next applied to these filtered data to obtain the complex analytic representation of each epoch. The angle and absolute magnitude of these complex time-series provided the instantaneous phase and amplitude of the $\delta$-band and $\beta$-band at each time point of each epoch. The $\beta$-band amplitude epochs were baseline corrected by subtracting the mean amplitude over the baseline period (after excluding the initial 100ms period which contained edge artifacts) from the amplitude at each time point over the epoch.

The Push-evoked and Release-evoked $\beta$-amplitudes were obtained by averaging the epoched $\beta$-band time-series relative to the time on each trial of the Push acceleration peak and the Release acceleration peak respectively after subtracting the $\beta$-amplitude at this reference point. Phase angle statistics were computed using CircStat (Berens 2009), a circular statistics toolbox implemented for MATLAB.

\subsection{Electrodes of interest}

Due to the putative confounds from differences between the contexts (see Section 1), we focused on the motoric demand common to both contexts, namely, to physically press a button on each trial. We, therefore, restricted our primary analysis of both contexts to EEG dynamics over the sensorimotor cortex contralateral and ipsilateral to the executed action (Fig. 1B) (i.e., 
representing the selected and non-selected action respectively). For all our EEG analyses, data from left and right hand trials were collapsed over corresponding electrodes contralateral to the moving finger (similarly for ipsilateral and medial electrodes), with electrodes over the left (right) hemisphere corresponding to contralateral (ipsilateral) electrodes respectively.

\section{Results}

\subsection{General behavior}

The general behavior of participants in both contexts indicated a high compliance with task instructions. In the Free context, the mean inter-response interval was $6618 \pm 136 \mathrm{~ms}$ (mean \pm s.e.m.) and only $1.6 \pm 0.6 \%$ of these intervals were shorter than $4 \mathrm{~s}$. The mean proportion of left $(49.3 \%)$ and right index finger button-presses (50.7\%) was also closely matched. In the Instructed context, the mean response accuracy was $97.7 \pm 0.5 \%$ consistent with the simplicity of the task. All subsequent analyses focused on actions in the Free context preceded by an interresponse interval greater than $4 \mathrm{~s}$ and correct trials in the Instructed context with responses times shorter than 1s (see section 2.6).

\subsection{Inter-hemispheric dynamics differ between contexts before the movement}

For actions to differ between the Free and Instructed contexts, a prerequisite is a difference in how those actions were neurally generated. Therefore, we first evaluated the event related potentials (ERPs) in the period immediately prior to and following movement onset. 

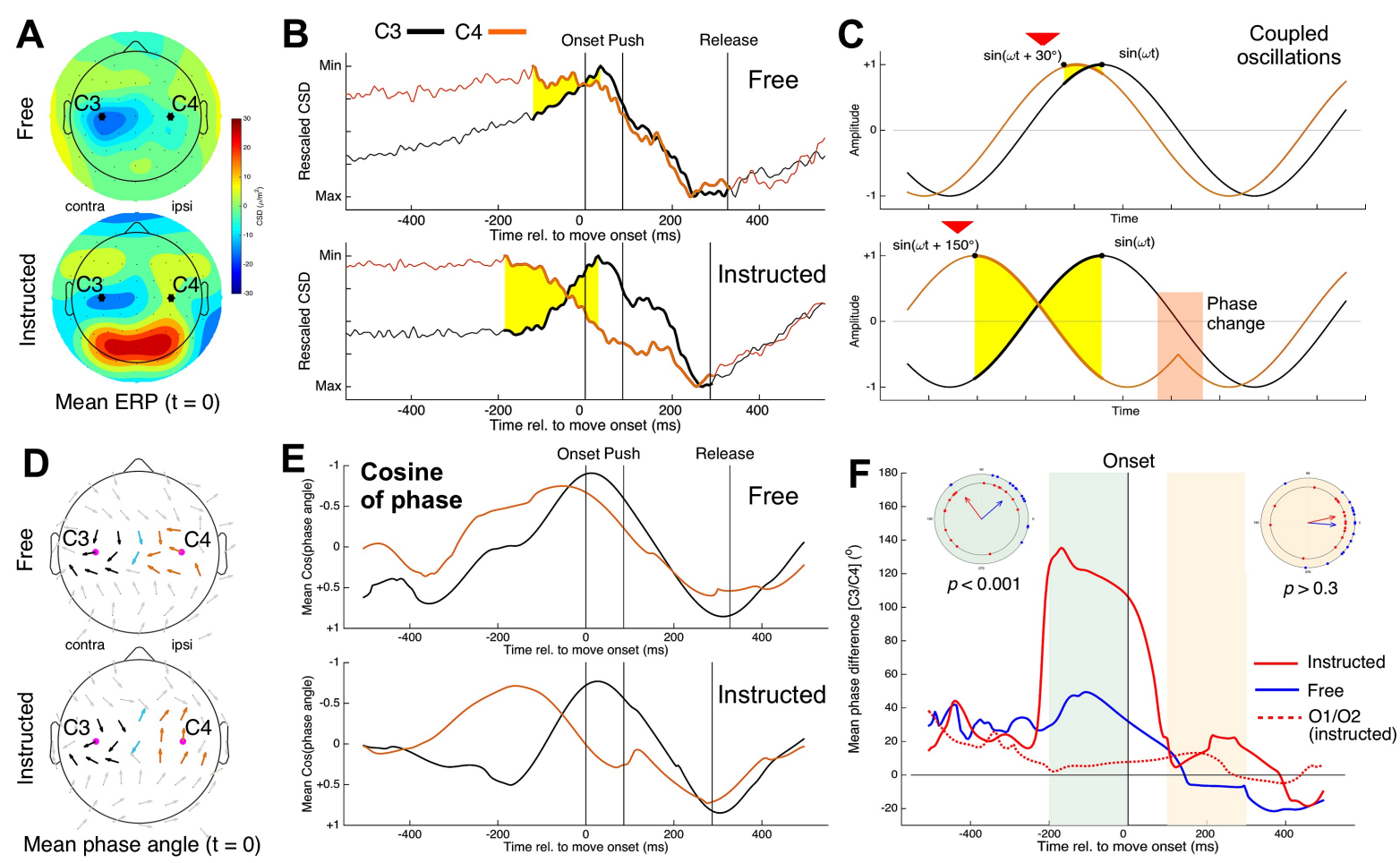

Figure 2. Interhemispheric ERP dynamics. (A) The topographic distribution of spatially filtered mean ERP amplitudes (Current Source Densities (CSD)) at movement onset. (B) ERPs at $\mathrm{C} 3$ and $\mathrm{C} 4$ were rescaled to have the same minimum/maximum values. Vertical lines indicate movement events. The duration between minima of C4 and C3 (yellow shading) was longer in Instructed context. (C) Hypothetical sine waves with coupled phases. Mismatch duration (yellow shading) is shorter for phase difference of $30^{\circ}$ (upper panel) than $150^{\circ}$ (lower panel). Phase difference of $150^{\circ}$ is shown as changing to $30^{\circ}$ via a 'reset'. (D) Instantaneous $\delta$-band phase angles at movement onset (colors distinguish contra/ipsi/medial electrodes). (E) Mean cosine of instantaneous $\delta$-band phase angles at C3 and C4. (F) Mean phase difference between $\mathrm{C} 3$ and $\mathrm{C} 4$. The dotted line indicates the mean phase difference between $\mathrm{O} 1$ and O2. Light green area indicates $[-200 \mathrm{~ms}, 0 \mathrm{~ms}]$, and orange area indicates $[+100 \mathrm{~ms},+300 \mathrm{~ms}]$. Mean phase angles for each period are shown in colored discs, dots correspond to mean angles for individual participants. 
<MOVIE 1 here $>$

Movie 1. A visualization of $\boldsymbol{\delta}$-band phase angle changes. The movie shows the changes in mean instantaneous $\delta$-band phase angles at each electrode, after initial explanatory frames. The phase angle is equal to the angle subtended by the arrow and the horizontal. Red arrows are phase angles in the Instructed context at the electrodes of interest (light orange arrows show phase angles at other electrodes for completeness). Blue (and cyan arrows) indicate phase angles in Free context. To highlight periods of interest, a yellow background is shown for the period [200, 0ms] relative to movement onset, and a gray background for [0,100ms]. As evident, the phase angles over contralateral electrodes are similar in both contexts but diverge substantially over the ipsilateral electrodes followed by a "reset". 
At movement onset in both contexts, the mean ERPs showed a focal negativity over central electrodes contralateral to the moved finger that was absent at homologous ipsilateral electrodes (Fig. 2A, S1). This spatial asymmetry in ERP amplitudes was consistent with expected motor activity lateralization before a unilateral index finger action. However, the arithmetic difference in ERP amplitudes at contralateral/ipsilateral locations masks their relative dynamics. When we rescaled the ERPs at representative contralateral and ipsilateral sensorimotor electrodes ( $\mathrm{C} 3$ and $\mathrm{C} 4)$ to equalize their minimum and maximum amplitudes, the dynamic rise and fall of these ERPs over time revealed a qualitative difference between contexts (Fig. 2B). At contralateral C3, the mean ERP reached its minimum value (i.e., peak negativity) shortly after movement onset in both contexts (Free: $+35 \mathrm{~ms}$, Instructed: $+30 \mathrm{~ms})$. Nonetheless, at ipsilateral C4, the corresponding ERP minimum in the Instructed context $(-185 \mathrm{~ms})$ was reached $65 \mathrm{~ms}$ earlier than in the Free context $(-120 \mathrm{~ms})$. Although this timing difference was seemingly specific to the ERPs at C4, when considered in conjunction with C3, the ERPs at $\mathrm{C} 3 / \mathrm{C} 4$ resembled coupled oscillations with a sustained phase difference closer to $0^{\circ}$ in the Free context but closer to the diametrically opposite value of $180^{\circ}$ in the Instructed context (Fig. 2C).

We statistically evaluated this descriptive formulation of ERP dynamics as coupled oscillations using the phase relationship of $\delta$-band oscillations (2-4Hz) at C3 and C4 (Fig. 2D). Oscillations in the $\delta$-band have been implicated in volitional selection in other tasks (HamelThibault et al. 2016). However, in the current study, these oscillations were of more pragmatic relevance due to their low frequency, which approximated the frequency of the ERP amplitude changes at $\mathrm{C} 3 / \mathrm{C} 4$. A simple demonstration of this (approximate) equivalence between $\delta$-band phase and ERP dynamics is the similarity between the ERP dynamics in Fig. $2 \mathrm{~B}$ and the mean cosine of the $\delta$-band phase angles at $\mathrm{C} 3$ and $\mathrm{C} 4$ in Fig. 2E. The cosine (rather than sine) is 
shown as the phase angle at contralateral C3 was close to $180^{\circ}$ at movement onset in both contexts.

In the Instructed context, the $\delta$-oscillations at $\mathrm{C} 3$ and $\mathrm{C} 4$ showed a phase difference closer to $180^{\circ}$ (rather than $0^{\circ}$ ) over a sustained $200 \mathrm{~ms}$ period before movement onset (Fig. $2 \mathrm{~F}$ ). Over the same $200 \mathrm{~ms}$ pre-onset interval, the mean phase difference between $\mathrm{C} 3$ and $\mathrm{C} 4$ in the Free context $\left(42^{\circ} \pm 20.90^{\circ}\right.$; mean $\left.\pm 95 \% \mathrm{CI}\right)$ remained closer to $0^{\circ}$ and was significantly lower than in the Instructed context $\left(125^{\circ} \pm 52.12^{\circ}\right)$ [Watson-Williams test, $F(1,34)=13.94, p<$ 0.001]. In contrast, over the $[+100 \mathrm{~ms},+300 \mathrm{~ms}]$ period following movement onset, the mean phase difference between $\mathrm{C} 3 / \mathrm{C} 4$ was close to $0^{\circ}$ in both contexts (Free: $-4.59^{\circ} \pm 22.17^{\circ}$; Instructed: $\left.13.30^{\circ} \pm 29.94^{\circ}\right)$ and not significantly different [Watson-Williams test, $F(1,34)=$ $1.04, p>0.3]$.

To control for the potentially spurious origin of these inter-context differences, we additionally evaluated the putative confounding role of the spatially oriented visual stimuli in the Instructed context. In this context, the arrow stimulus on each trial could plausibly evoke ERPs lateralized relative to the arrow's (left/right) orientation rather than the (left/right) index finger action that it instructed. Even if such stimulus-evoked ERPs were to originate at occipital sources, they might nonetheless drive the phase differences at $\mathrm{C} 3 / \mathrm{C} 4$ due to volume conduction. However, incompatible with such spurious effects, the mean phase difference in $\delta$-phase at occipital electrodes (contralateral) $\mathrm{O} 1$ and (ipsilateral) $\mathrm{O} 2$ remained close to $0^{\circ}$ before and after movement onset in the Instructed context (dotted line, Fig. 2F). Furthermore, over the [-200ms, $0 \mathrm{~ms}]$ interval, the mean phase difference between $\mathrm{O} 1$ and $\mathrm{O} 2\left(5.55^{\circ} \pm 7.59^{\circ}\right)$ was significantly lower than the phase difference between C3 and C4 over this period [Watson-Williams test, $F(1,34)=34.48, p<0.001]$. For completeness, we additionally evaluated the mean $\delta$-phase 
difference over the $[-200 \mathrm{~ms}, 0 \mathrm{~ms}]$ period at contralateral-ipsilateral electrode pairs over the scalp (Fig. 3; please, also see the Movie 1 for an animation of instantaneous phase angle changes across the scalp). The inter-hemispheric (absolute) phase differences were uniformly low across the scalp in the Free context but phase differences greater than $90^{\circ}$ were focally present only over $\mathrm{C} 3 / \mathrm{C} 4$ (and its immediate neighbors) in the Instructed context. This focally high phase difference at $\mathrm{C} 3 / \mathrm{C} 4$ was significantly greater than the corresponding value at all other non-neighboring electrodes (Fig. 3B) and was inconsistent with a spurious origin linked to stimulus orientation.

In summary, ERP amplitudes over the central electrodes showed a similar pre-movement lateralization in the Free and Instructed contexts. However, the inter-hemispheric ERP dynamics in the two contexts (expressed in terms of $\delta$-phase differences) were near theoretically opposite extremes of the phase angle scale (i.e., $0^{\circ}$ and $180^{\circ}$ ). At first glance, this striking difference in sensorimotor dynamics was evidence that the binary choice between the same action alternatives in the Free and Instructed contexts had differing consequences for the motoric representations of these actions. The behavioral relevance of this difference was unclear as the pre-movement ERP amplitude and local dynamics at contralateral C3 were highly similar in the Free and Instructed contexts. These dynamics could, for instance, be an incidental aftereffect of how decision evidence accumulated in the motor system in the two contexts (Selen, Shadlen, and Wolpert 2012; Thura and Cisek 2014), that is, indicating that both actions were equally preferable in the Free context (low phase difference) but that one was preferred over the other in the Instructed context (high phase difference). To assess the behavioral relevance of the inter-context differences in ERP dynamics, we next evaluated the kinematics of the executed actions in the two contexts. 

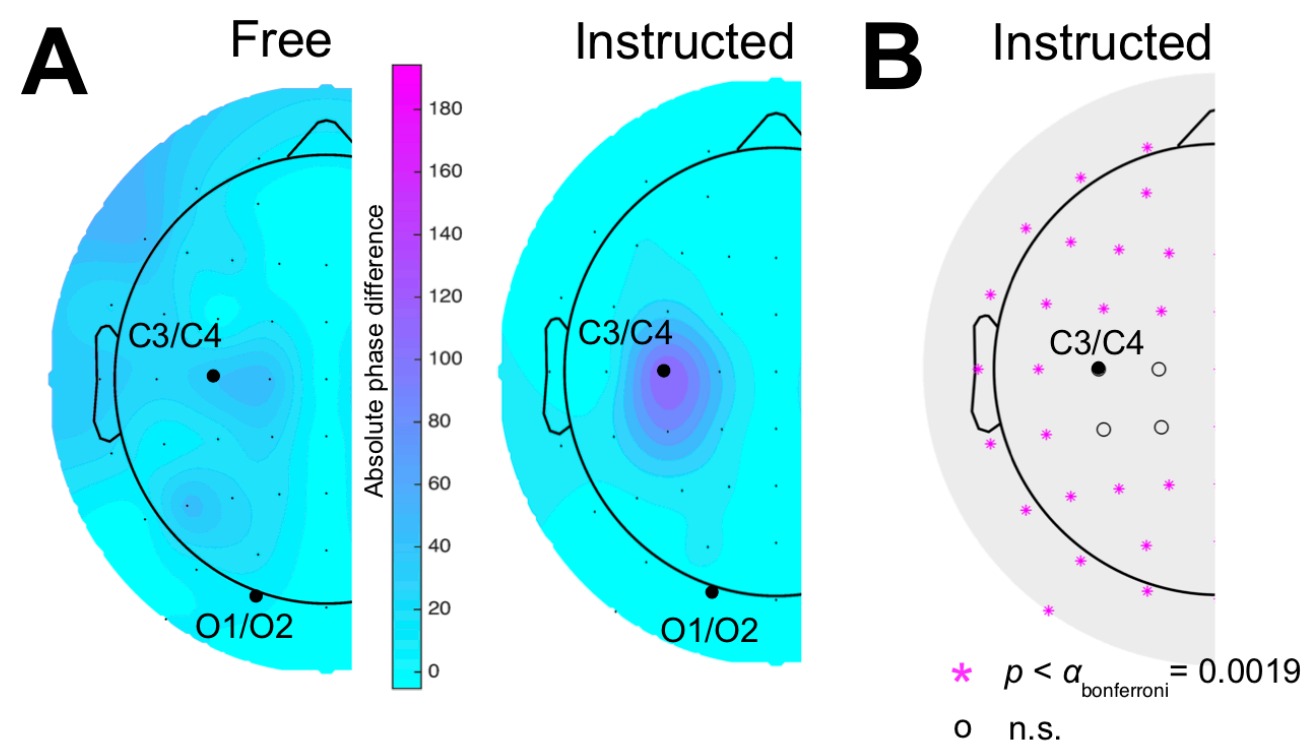

Figure 3. Phase differences across the scalp. (A) The absolute mean phase differences between each contralateral electrode and its ipsilateral homologue averaged over [-200ms,0ms] in the two contexts. (B) The distribution of electrode pairs in the Instructed context with a mean phase difference that was significantly different from the mean difference at $\mathrm{C} 3 / \mathrm{C} 4$ [corrected for multiple comparisons]. No electrode pair showed a corresponding statistically significant difference in the Free context. 


\subsection{The timing of the button release movement differs between contexts}

To assess the influence of the Free and Instructed contexts on behaviour, we used the accelerometer recordings to distinguish the two movements involved in pressing the button, namely, the index finger's rapid flexion to push the button down and its subsequent extension to release the button (see Section 2.6.5). On each trial, the Push and the Release movement could each be associated with a separate (absolute) acceleration peak (Fig. 4A, upper panel). The acceleration peak uniquely linked to each Push and each Release movement was described by the peak's magnitude and its latency relative to movement onset.

The mean latency of the Push and Release movements (i.e., acceleration peaks) (Fig. 4B, left panel) were significantly modulated by context [analysis of variance (ANOVA), Context $\{$ Free, Instructed $\} \times$ Movement $\{$ Push, Release $\}$, Context*Movement: $F(1,17)=25.57, p<$ 0.001; Context: $F(1,17)=27.05, p<0.001$; Movement: $F(1,17)=277.63, p<0.001]$ but the corresponding mean acceleration magnitudes (Fig. 4B, right panel) were not [ANOVA, Context*Movement: $F(1,17)=0.08, p=0.78$; Context: $F(1,17)=4.31, p=0.054$; Movement: $F(1,17)=52.11, p<0.001]$. The mean latency of the Push movement was indistinguishable between contexts $[t(17)=-0.30, p>0.7]$ but the mean Release latency in the Instructed context $(287.14 \pm 11.19 \mathrm{~ms}$; mean \pm within-subject s.e.m. (Cousineau 2005)) was significantly smaller than in the Free context $(327.26 \pm 15.37 \mathrm{~ms})[t(17)=5.15, p<0.001]$. This inter-context difference in Release latency was present for 17 of our 18 participants and an example of this modulation for one participant is shown in Fig. 4A (lower panels). 

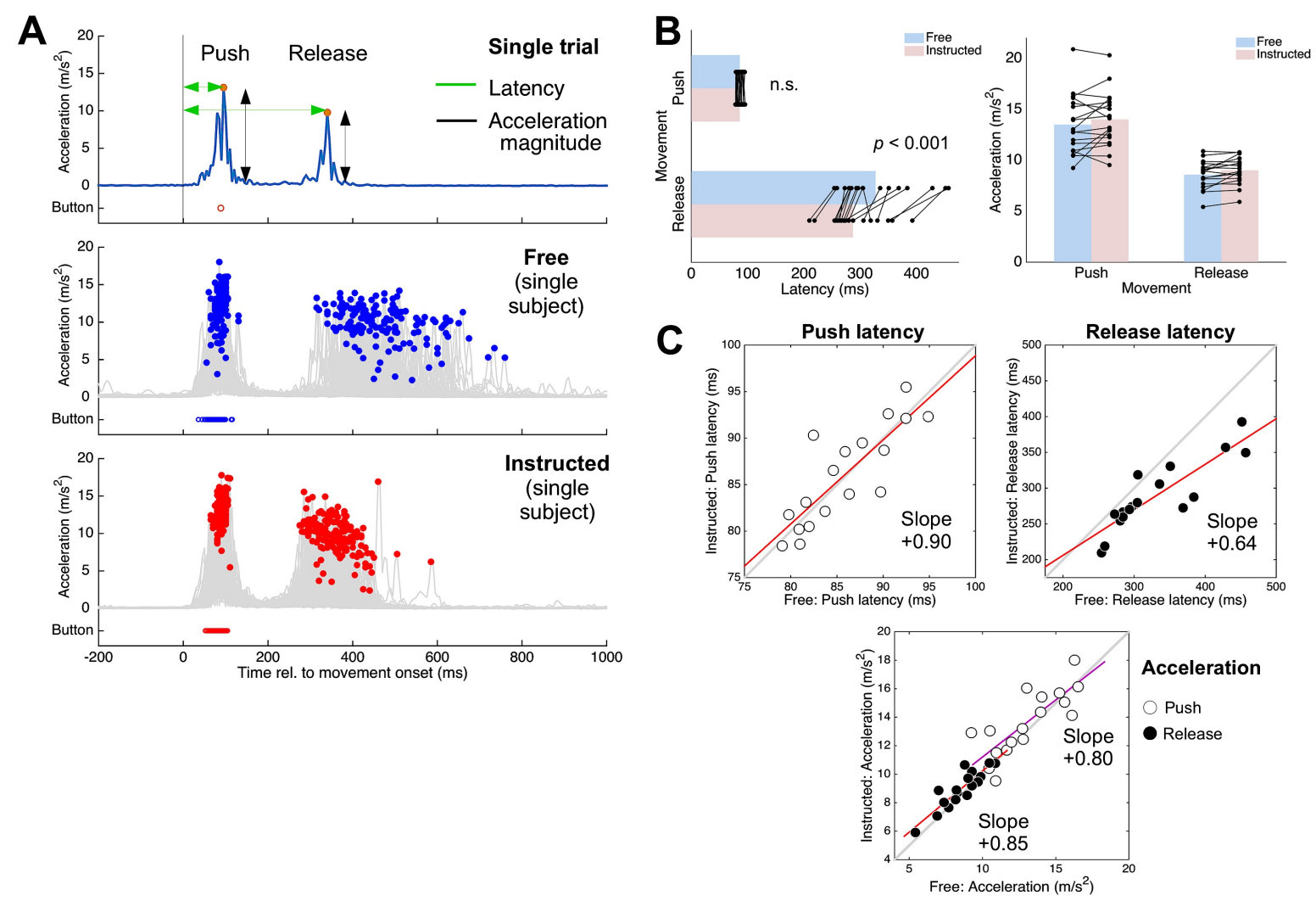

Figure 4. Movement kinematics. (A) Absolute acceleration of Push and Release movements on a single trial (first panel), and on all trials from one representative subject (lower panels). Colored dots indicate acceleration peaks (Release peaks occurred earlier in the Instructed context) (B) Mean latencies (left panel) were significantly modulated by context but mean accelerations (right panel) were not. Each connected pair of black dots represents values for the same participant (C) Between-context correlations in Push latency (upper left), Release latency (upper right), Push (open dots) and Release accelerations (filled dots) (lower). Colored lines indicate the linear relationship (best least-squares fit) between corresponding variables in the Free (x-axis) and Instructed contexts (y-axis). The unit slope line is shown in gray. 
To clarify these inter-context kinematic differences, we next assessed the statistical association of the Push and Release kinematics between contexts, i.e., whether the kinematics in one context were informative about corresponding properties in the other context. Even though the mean values of the Push and Release kinematics were similar between contexts, the factors that determined these kinematic properties might be context-specific. This possibility would predict a weak statistical association of the kinematics between contexts. However, inconsistent with this possibility, the Push kinematics in the Free and Instructed contexts were robustly correlated (i.e., between contexts) [latency: $r(17)=0.83, p<0.001$; acceleration: $r(17)=0.86, p$ $<0.001$ ] and so were the Release kinematics [latency: $r(17)=0.87, p<0.001$; acceleration: $r(17)=0.89, p<0.001]($ Fig. 4C) .

The between-context correlations for the Push and Release latency had similar correlation coefficients ( $r=0.83$ and 0.87 . respectively) but the scaling of their respective linear relationships was different. The slope of the linear relationship of the Push latency in the two contexts was equal to +0.90 (i.e., close to 1) but the slope for the Release latency had a lower value of +0.64 (Fig. $4 \mathrm{C}$, upper row). This lower value was not a general peculiarity of the Release action as the slope for Release acceleration had a comparatively higher value $(+0.85)$ that was closer to the slope for the Push acceleration $(+0.80)$ (Fig. 4C, lower row). The lower valued slope for the Release latency highlighted a subtle but critical feature of the $\sim 40 \mathrm{~ms}$ mean latency difference between contexts. The relationship between Release latency in the two contexts (denoted by $L_{\text {Instr }}$ and $L_{\text {Free }}$ ) could hypothetically have taken the form $L_{\text {Instr }} \approx L_{\text {Free }}$ (i.e., slope equal to +1$)$ rather than $L_{\mathrm{Instr}} \approx(0.64) L_{\text {Free. }}$ This possible alternative would have implied that the mean difference between $L_{\text {Instr }}$ and $L_{\text {Free }}$ was primarily due to an additive "speedup" (or "slowdown") in one context relative to the other (see Fig. S3 for one example). By ruling out 
such a purely additive basis for the Release latency difference, these data constrain the possible origins of this difference to factors tied to both contexts rather than independent factors specific to each context. One such non-independent origin of the Release latency differences that is common to both contexts was the Push action itself.

In the statistical tests above, the Push and Release actions were treated as independent actions even though, by definition, the Release always followed the Push action and the Push and Release involved moving the same index finger in quick succession on each trial. Despite this apparent interdependence of the Push and Release actions, their respective kinematics within the same context were uncorrelated both within the Free context [latency: $r(17)=-0.38$, $p=0.12$; acceleration: $r(17)=0.08, p=0.74]$ and within the Instructed context [latency: $r(17)$ $=-0.23, p=0.36$; acceleration: $r(17)=0.13, p=0.62]$. This behavior-to-behavior relationship was, however, inadequate to account for another important form of Push-Release dependence. On each trial, the Release not only followed the physical execution of the Push action but also the diverse neural processes that the Push action itself evoked, for example, the somatosensory afferents generated by the movement of the "pushing" index finger itself and the finger's extended physical contact with the button until the Release. We next assessed the dependence of the Release on these Push-evoked neural processes, and if this dependence differs between contexts in a manner that might modulate the latency of the Release action. 


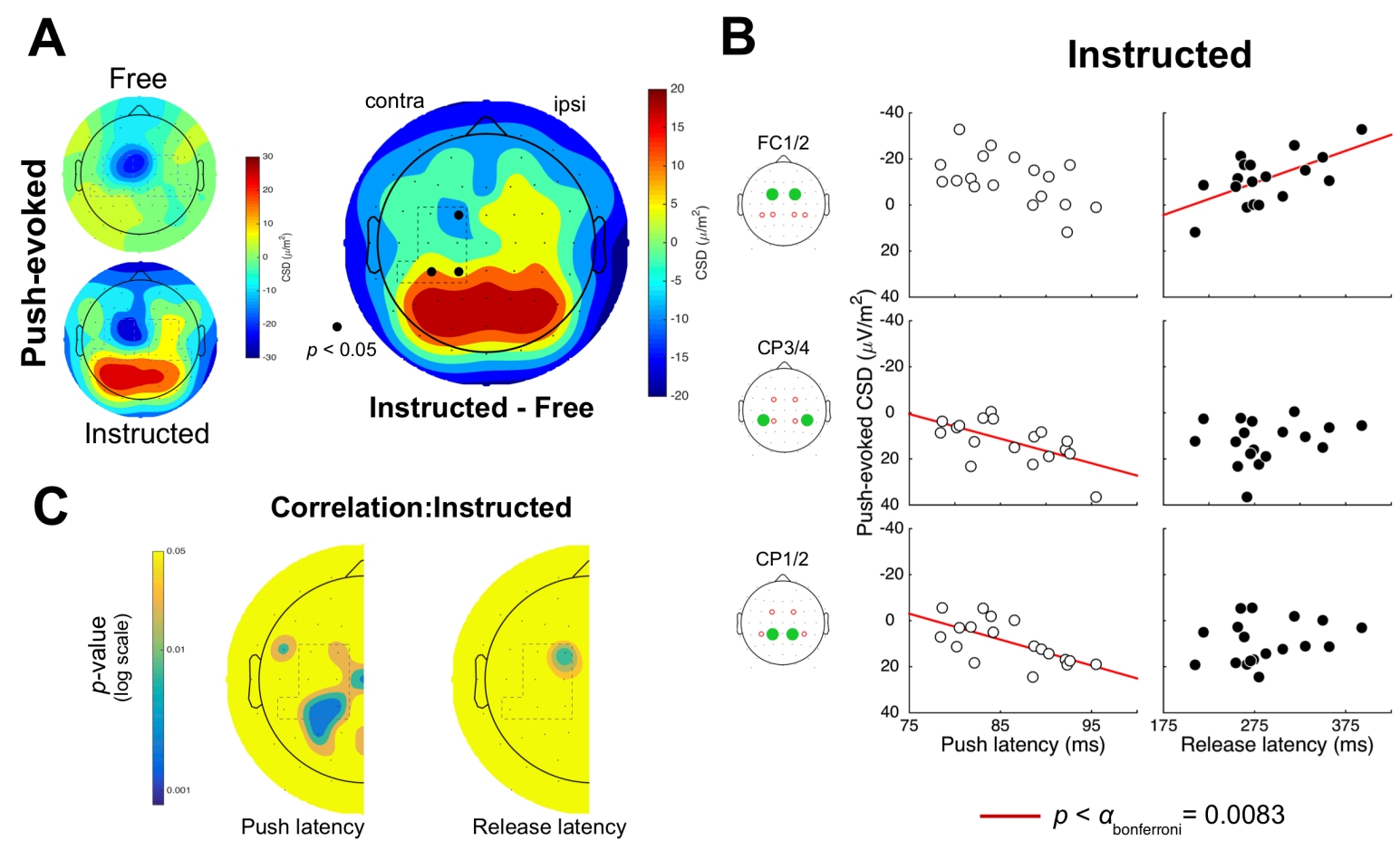

Figure 5. Movement-evoked ERPs. (A) The topographic distribution of mean Push-evoked ERPs in each context and their difference. (B) Correlations of Push-evoked ERPs to Push latencies (left column) and Release latencies (right column) for Instructed context. (C) Topographic distribution of p-values (log scaled) of correlations between Push-evoked ERPs and Push latencies (left) and Release latencies (right) for the Instructed context. 


\subsection{Push-evoked ERPs predict Release latency in the Instructed, but not Free, context}

The accelerometer recordings acquired in our experiment did not allow a precise characterization of the contact between the finger and the button. In its place, the Push and Release latency that were estimated on every trial were used to compute the ERPs evoked by these movements.

We first evaluated whether there was any modulation of the mean Push- and Releaseevoked ERP amplitudes (averaged over a 70ms window following each movement's acceleration peak) by context at each of the 7 contralateral electrodes of interest [ANOVA, Context $\{$ Free, Instructed $\}$ x Movement $\{$ Push, Release $\}$, full statistical details in Table A.1 (upper)](also see Fig. S2A, B). Context significantly modulated the movement-evoked ERPs at three electrodes - frontocentral FC1, centroparietal CP1, CP3 - but notably not at C3. Apart from this general modulation, the mean Push-evoked ERP amplitudes at these three electrodes also differed significantly between contexts (Fig. $5 \mathrm{~A}$ ) $[\mathrm{FC} 1: t(17)=2.87, p=0.01 ; \mathrm{CP} 3: t(17)=-$ 5.14, $p<0.001$; CP1: $t(17)=-3.48, p=0.003$ ]. The polarity of the Push-evoked ERPs at these electrodes (negative at $\mathrm{FC1}$, positive at $\mathrm{CP} 1, \mathrm{CP} 3$ ) and their lateralization properties (significantly lateralized at $\mathrm{FC} 1 / 2$, but not at $\mathrm{CP} 1 / 2$, CP3/4 (Table A.1 (lower), Fig. S2C)) further suggested that they had distinct neural generators. To incorporate the differing lateralization properties at these electrodes into our analysis, their contralateral and ipsilateral ERP amplitudes were averaged for further tests.

To assess the dependence of Release latency on these Push-evoked neural responses, we evaluated the statistical association between the bilateral Push-evoked ERPs at the three electrode-pairs (FC1/2, CP1/2, CP3/4) to the corresponding Push and Release latencies within the same context (Fig. 5B; full statistical information in Table A.2). In the Instructed context, 
the mean bilateral Push-evoked ERPs at CP1/2 and CP3/4 (but not FC1/2) were significantly correlated with mean Push latency while the ERP at FC1/2 (but not CP1/2, CP3/4) was significantly correlated with mean Release latency. Surprisingly, the corresponding Pushevoked ERPs in the Free context were not informative about either the Push or Release latency. For completeness, the topographic distribution of p-values of the ERP-movement correlations in the Instructed context for all electrode pairs is shown in Figure 5C.

The statistical association of the Push-evoked ERP at FC1/2 to the latency of the subsequent Release action, i.e., to be executed at a future time, confirmed the crucial distinction between the Push-evoked neural processes and the Push action's kinematics (to which the Release was not significantly associated). The divergence in ERP-behavior correlations at FC1/2 and $\{\mathrm{CP} 1 / 2, \mathrm{CP} 3 / 4\}$ was consistent with the diverse origins of the Push-evoked neural activity. The multiple brain-to-behavior associations in the Instructed context, together with their absence in the Free context was surprising since the Push and Release kinematics were robustly associated between contexts (as described in section 3.3).

The categorical absence of these associations both to the Push or Release latency in the Free context was prima facie evidence of a difference in sensorimotor organization from the Instructed context that might account for their Release latency differences. However, this inference about the Free context based on a statistical null effect might instead reflect the role of a confounding process. For example, the Free, but not Instructed, context required participants to estimate time-intervals of 4-8 seconds between consecutive button-presses. The neural mechanisms involved in time-estimation involving regions such as the supplementary motor area (SMA) and pre-SMA(Grondin 2010) might have distorted the Push-evoked ERPs at FC1/2 due to their spatial proximity. To obtain an alternative (non-ERP) perspective to disentangle 
these alternative possibilities, we evaluated the relationship of the Push and Release movements to a well-established higher frequency oscillatory signature of movement completion.

\subsection{Onset of $\beta$-power rebound is linked to different movements in the two contexts}

Spectral power in the $\beta$ band $(13-30 \mathrm{~Hz})$ has been shown to characteristically increase (or rebound) over the central electrodes following a movement, also known as Event-Related Synchronization (ERS), and has been attributed to an inhibitory reset of the primary motor cortex (Pfurtscheller and Lopes da Silva 1999; Jurkiewicz et al. 2006; Kilavik et al. 2013). This phenomenon suggested a way to relate the Release-related findings to the mechanisms of how an action was terminated. For this purpose, the "post-movement" dimension of the $\beta$-rebound was of particular relevance. Our decomposition of the actions to press a button into a Push and a Release action presented an ambiguity about which of the two movements would serve as the

reference movement for the "post-movement" $\beta$-rebound. In prior studies of the execution of self-initiated multi-movement sequences, the $\beta$-rebound has been reported to commence after the sequence's final movement rather than after each individual movement (Cassim et al. 2000; Alegre et al. 2004; Erbil and Ungan 2007; Cheyne 2013). A plausible prediction, based on these prior studies, was that the $\beta$-rebound would follow the Release movement in both contexts, namely, the final movement of the Push-Release sequence. 

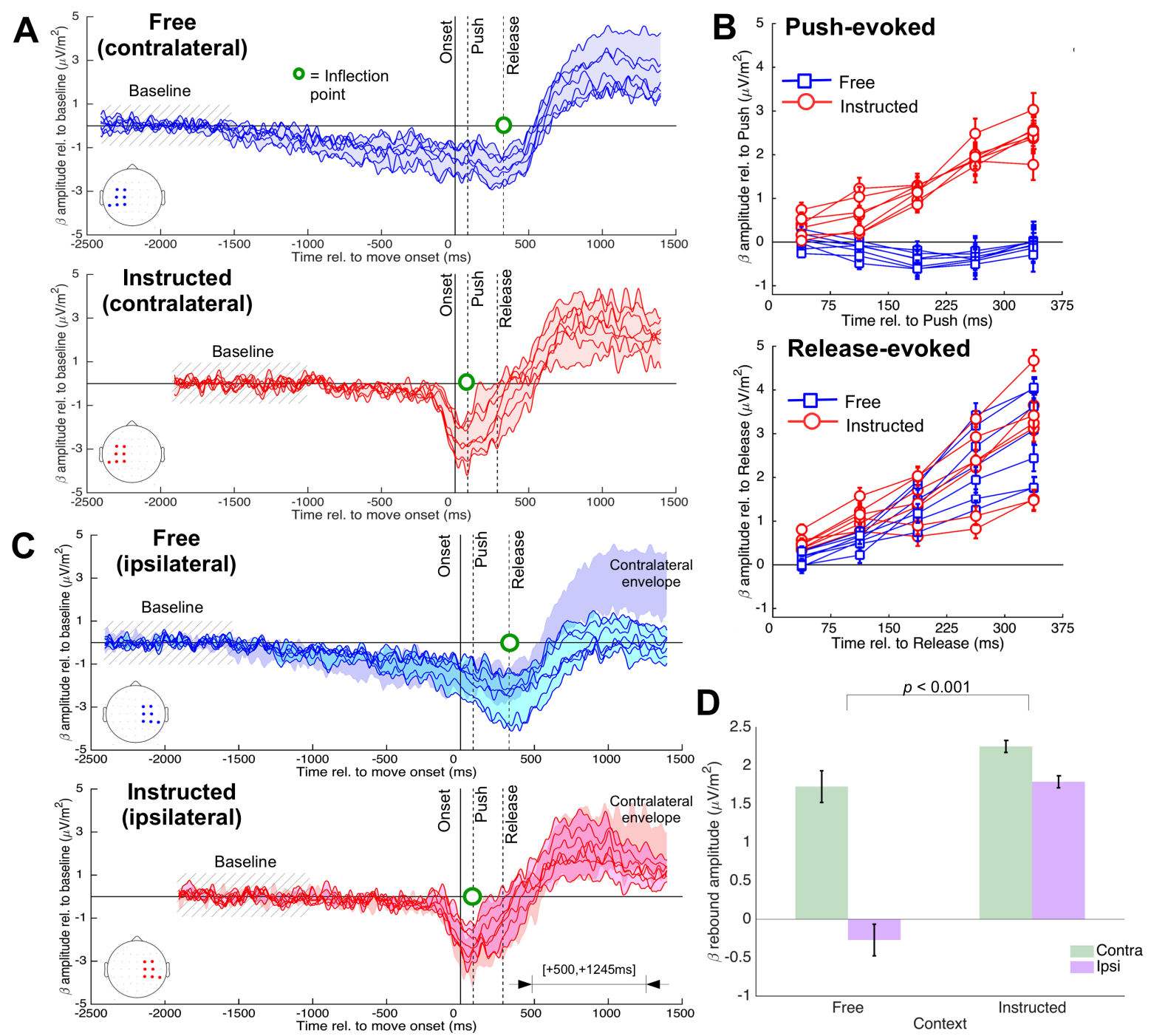

Figure 6. Context-dependence of $\boldsymbol{\beta}$-rebound. (A) Mean $\beta$-amplitude at contralateral electrodes relative to baseline (shading indicates amplitude envelope). The green circle marks the movement closest to the $\beta$-rebound's onset time. (B) Push-evoked (upper panel) and Releaseevoked (lower panel) changes in $\beta$-amplitude over $375 \mathrm{~ms}$ post-movement period ( $5 \times 75 \mathrm{~ms}$ bins). Error bars indicate within-subject s.e.m. (Cousineau 2005). (C) Mean $\beta$-amplitude at ipsilateral electrodes. Contralateral amplitude envelopes are shown as a reference. (D) Mean contralateral and ipsilateral $\beta$-amplitudes over the period $[+500 \mathrm{~ms},+1245 \mathrm{~ms}]$. 
In our data, a $\beta$-rebound was evident at all seven contralateral electrodes in the Free and Instructed contexts (Fig. 6A). The baseline-corrected power in the $\beta$-band exhibited a decrease prior to movement-onset in both contexts, with this decrease being more rapid and starting closer to movement onset in the Instructed context. The "rebound" occurred at a time point following movement-onset, where the power changes switched from decreasing continuously over time to increasing monotonically until a plateau value.

The timing of the contralateral $\beta$-rebound seemed to be yoked to the Release movement in the Free context but, remarkably, this rebound in the Instructed context was yoked to the Push movement, i.e., $\sim 241 \mathrm{~ms}$ earlier than in the Free context. We statistically confirmed this observation at all 7 contralateral electrodes by evaluating the context-dependent modulation of the mean Push-evoked and Release-evoked changes in $\beta$-amplitude over a $375 \mathrm{~ms}$ period (discretized into five $75 \mathrm{~ms}$ bins)(ANOVA, Context $\{$ Free, Instructed $\} \times$ Time $\left\{\mathrm{t}_{1}, . ., \mathrm{t}_{5}\right\}$, full statistical details in Table A.3) (Fig. 6B). Following the Push movement, the change in $\beta$ amplitude over time showed a significant modulation by context (Fig. 6B upper), but this contextual modulation was not statistically significant following the Release movement consistent with the rebound being underway in both contexts (Fig. 6B lower).

The contralateral and ipsilateral rebound amplitudes were closely matched in the Instructed context (Fig. 6C lower panel) but, in the Free context, the ipsilateral rebound amplitude did not exceed baseline levels (Fig. 6C upper panel). Over the period [+500ms, $+1245 \mathrm{~ms}$ ] following movement onset, the relationship of the mean contralateral $\beta$ amplitude (averaged across electrodes) to the corresponding ipsilateral amplitude was significantly modulated by context [ANOVA, Context $\{$ Free, Instructed $\} \times$ Laterality $\{$ Contra, Ipsi $\}$, 
Context*Laterality: $\mathrm{F}(1,17)=17.942, \mathrm{p}<0.001$; Context: $\mathrm{F}(1,17)=15.687, \mathrm{p}<0.005$; Laterality: $F(1,17)=23.426, \mathrm{p}<0.001]$ (Fig. 6D).

These findings provide a converging line of evidence to clarify the ERP-based findings above ((section 3.4) hinting at a categorical difference in how actions are terminated in the Free and Instructed actions. The onset of the $\beta$-rebound associated with the Push movement in the Instructed, but not Free, context was congruent with the presence of Push-evoked ERP-toRelease associations in the Instructed, but not Free, context. The categorical absence of these Push-related associations in the Free context was consistent with a different sensorimotor organization between contexts but not the role of spurious artifacts from time-estimation processes. The onset of the $\beta$-rebound specifically linked to the Release movement in the Free context, rather than simply being delayed relative to the Push movement, further affirmed the categorical nature of the sensorimotor differences between the Free and Instructed contexts. The prominent difference in lateralization of the $\beta$-rebound between contexts provided an independent validation of the context-dependent difference in inter-hemispheric ERP/ $\delta$-phase dynamics. If the $\beta$-rebound is an indicator of the resetting of "active" motor representations then the above findings suggest that the motor representations of both action choices (contra and ipsi) were concurrently active in the Instructed context but only the selected action (only contra) was motorically represented in the Free context.

\section{Discussion}

In decision terms, our experiment required participants to develop a preference for one of two alternative courses of action on each trial and report this preference by executing a pre- 
designated action (i.e., an index finger button press). However, these decisions did not occur in isolation but in the context of multiple experimental features used to constrain the information used to develop the preference, i.e., specified by an explicit instruction (Instructed context) or in the explicit absence of an instruction (Free context). Here we found multiple lines of evidence that there was a structured difference in how actions were executed in the Free and Instructed contexts even when reporting the same preference.

It is worth carefully considering how our findings narrowly related to the kinematic minutia of index finger movements and associated motor system dynamics could relate to broader considerations about volition and free choice. A first concern is whether the action differences between contexts could be attributed to the information constraints (presence/absence of instructions that modulated volition's role) or instead to the factors involved in implementing these constraints (e.g., stimuli, inter-response timing, reward differences, etc.).

\subsection{Chronometry of action termination}

A novelty of our study was the decomposition of the button-press event into its action elements, namely, a Push and a Release. Unlike the Push action that was required to report the cognitive choice (i.e., either freely selected or instructed), the Release had no direct functional role in the cognitive task and was incidental to the use of index finger button presses in our experiment rather than another action, such as a saccade. Nevertheless, a salient and surprising finding was that the Release movement in the Instructed context was executed $\sim 40 \mathrm{~ms}$ earlier than in the Free context, while all other measured aspects of the kinematics were unaffected by context. 
The two contexts had another chronometric difference closely linked to the Release action. Congruent with the Release latency difference, the contralateral $\beta$-rebound also had an earlier onset in the Instructed context than in the Free context but with a timing difference ( $241 \mathrm{~ms})$ that was six times the size of the Release latency difference. This large timing difference was a consequence of the yoking of $\beta$-rebound's onset to the Push movement in the Instructed context but to the Release movement in the Free context.

In an isolated EMG study of right index-finger flexion movements, Obhi and Haggard (2004) found that the electromyogram (EMG) at the first dorsal interosseus muscle of the right hand was slightly larger and sustained for marginally longer when the flexion was self-initiated than when it was instructed. Although suggestive of a similarity to our findings, it is currently unclear whether those EMG duration differences and the Release timing differences in our study are consequences of the same physiological mechanism. In that study, Obhi and Haggard (2004) postulated that the EMG difference that they observed might be a consequence of inter-context differences in motor preparation. Preparation mechanisms might certainly be organized differently in the two contexts (Obhi, Matkovich, and Gilbert 2009; Hughes, Schutz-Bosbach, and Waszak 2011; Schurger, Sitt, and Dehaene 2012; Wang et al. 2017). Yet, a variety of other explanations might also account for the Release latency difference. However, the relationship of the $\beta$-rebound to the Release action places a strong constraint on possible explanations of the inter-context chronometric difference.

We now consider some examples of seemingly plausible models of the Release latency differences that are nonetheless incompatible with the timing properties of the $\beta$-rebound. One possibility is that the Push-then-Release sequence might be executed as a single action unit having a shorter overall duration in the Instructed context than in the Free context (Fig. 7A). 
Another class of possible mechanisms is suggested by analogy to sequential sampling models for Response Times (Roger Ratcliff 2008; O’Connell et al. 2018). In this framework, the Release action might be initiated when a decision variable linked to the Push action reaches a designated decision criterion. The Release latency differences might therefore arise because this decision criterion was set lower in the Instructed context than in the Free context (Fig. 7B); or because the decision variable increased more rapidly in the Instructed context relative to the Free context (Fig. 7C). All the above models assume that actions in both contexts share a common implementation having the same continuously varying parameters (e.g., action duration, decision criteria, accumulation rate, etc.). Accordingly, the Release latency difference is explained as resulting from differences in the parameter settings between contexts. This assumption about shared continuous parameters was, however, incompatible with the categorical difference in $\beta$-rebound timing between contexts, i.e., linked to the Push movement in the Instructed context but to the Release movement in the Free context. This incompatibility would be absent if the $\beta$-rebound were similarly structured in both contexts, i.e., linked to the Push or to the Release in both contexts, or if the $\beta$-rebound had onset differences between contexts without being linked to either Push or Release action.

Based on physiological considerations, we propose a novel model to reconcile this timing relationship between the Release action and the $\beta$-rebound. The central idea is that the Release action might be initiated based on different kinds of information in the two contexts that are generated at different times during action execution. 


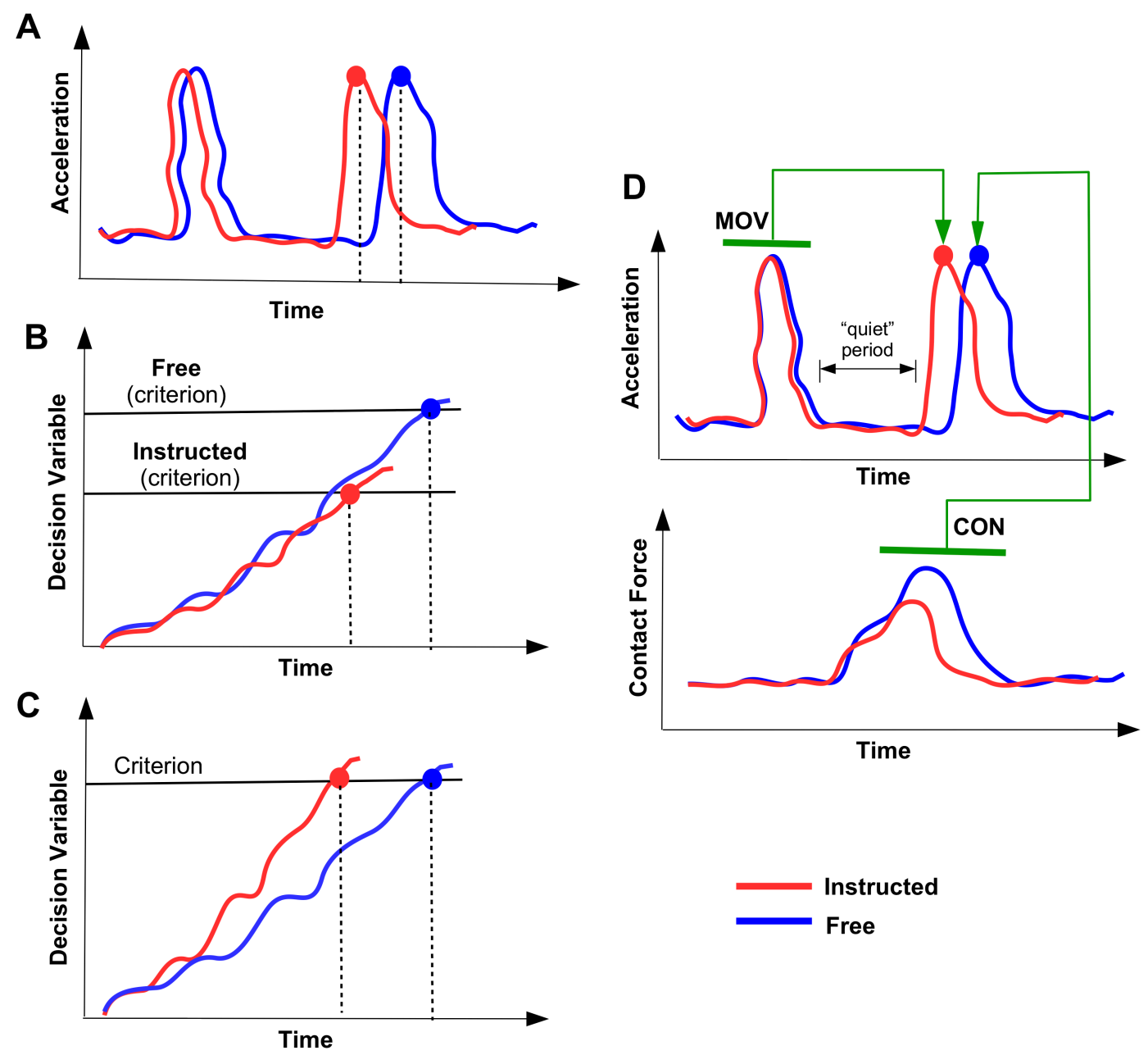

Figure 7. Release timing models. (A) Differences in overall movement timing in Free (blue) and Instructed context (red). (B) Difference in post-Push decision criteria between contexts. Colored dots indicate time at which decision criterion is met. (C) Difference in rate of increase in post-Push decision variable. (D) Information driving Release is indicated by thick green lines and arrows: Acceleration changes during Push movement (MOV) (upper panel), and contact forces during post-Push "quiet" period (CON) (lower panel). 


\subsection{Action termination: Dual criterion model}

Our accelerometer recordings index the small, rapid movements of the index finger but do not measure the contact forces between the index finger and the surface of the button. In a typical accelerometer trace of movements on a trial, there is a readily noticeable "quiet" period that is free of acceleration changes in between the Push and Release acceleration peaks (Fig. 4A). This period corresponds to the interval when the neural forcing of the finger's movement leads to continued application of pressure by the finger on the button's surface, even when no further downward movement of the button is possible (Oulasvirta, Kim, and Lee 2018). As a result, the contact force and associated compression of the finger's soft tissue possibly reaches a maximum value at some point during this "quiet" period before reducing with the onset of the Release.

The above mechanical considerations suggest that there might be two relatively independent criteria for the sensorimotor system to determine when the button was successfully pressed: (i) the time at which the neural generation of muscular forces no longer produced a corresponding finger movement and (ii) the time at which the contact force reached a suitably high value. Evaluating each criterion requires information that is generated at different periods of time relative to movement onset. Assessing the first, movement-related criterion (henceforth, MOV) would involve integrating efferent motor information with proprioceptive feedback about the moving index finger within a control model (Miall and Wolpert 1996; M Desmurget and Grafton 2000). However, assessing the second contact-related criterion (henceforth, CON) would depend on tactile percepts generated by the contact between the index finger and button during the "quiet" period.

Based on these simplistic considerations, we propose a model, which we refer to as the Dual criterion model. According to this model, neural processes are configured to initiate the 
Release action primarily based on the MOV criterion in the Instructed context and primarily based on the CON criterion in the Free context (Fig. 7D). The MOV criterion is assumed to be successfully satisfied at an earlier time than the CON criterion since the movement-related information is available before the contact-related information reaches its peak intensity. The relative timing of the MOV and CON criteria are predicted to produce the observed difference in Release latency in the two contexts. Specifically, the MOV criterion is assumed to be satisfied at a time point during the acceleration changes associated with the Push movement itself, with a short refractory delay before the Release can begin. However, the CON criterion is assumed to be satisfied during the "quiet" period enabling the Release to follow quickly after. The $\beta$-rebound's onset is assumed to be related to the neural changes triggered when these criteria are met. The apparent linkage to the Push/Release movements is an indicator of the time period when these criteria are satisfied. The onset of the $\beta$-rebound would therefore be closely linked to the Push movement in the Instructed context while being more closely linked to the Release movement in the Free context.

A crucially relevant body of evidence supporting the existence of two such distinct modes for controlling fine finger movements comes from studies where tactile feedback was blocked by anesthetizing the fingertips. Touch typing (without visual feedback) by expert typists with anesthetized finger tips produced increased spatial errors in finger placement but did not influence movement kinematics or keystroke timing (Gordon and Soeehting 1995; Rabin and Gordon 2004), consistent with a movement-based control of these rapid finger movements. By contrast, in studies of synchronized finger tapping to periodic auditory tones, anesthesia disrupted movement timing (Aschersleben, Gehrke, and Prinz 2001). This timing-related disruption supports the hypothesis that synchronization is achieved by timing finger movements 
in order to coordinate the timing of the resulting tactile percept with the auditory percept (Aschersleben 2002; Goebl and Palmer 2008). This form of touch-governed movement control is consistent with a contact-based criterion.

Another supporting line of evidence has to do with the neural basis for movement awareness. Based on evidence from direct cortical stimulation of neurosurgical patients, Desmurget and Sirigu (2009) emphasize the differential neural basie of movement awareness and the intention/urge to move. They specifically suggest a key role of the posterior parietal cortex in movement awareness and of the premotor cortex in the urge to move. Superficially consistent with this proposal, in our study, Push-evoked ERPs were correlated with movement latency in the Instructed context where a key role for the MOV criterion was hypothesized. The Push-evoked ERPs at the centro-parietal electrodes were associated with the Push-latency (consistent with a monitoring of ongoing movements) while the Push-evoked ERPs at FC1/2 were associated with the latency of the upcoming Release movement (consistent with the urge to initiate the Release action). This frontal/parietal distinction in ERP-movement correlations was also evident across the scale (Fig. 5C). Even though the Push action's kinematics was closely matched between contexts, these ERP-movement-related correlations were absent for the Pushevoked ERPs in the Free context where a key role for the CON criterion was hypothesized.

In summary, the Dual criterion model accounts for the difference between the contexts in Release latency, the discontinuous timing of the $\beta$-rebound, as well as the ERP-behavior correlations. Although based on assumptions about the source of sensory information, this model's central predicted consequence is a difference in the neural organization to terminate actions in the Free and Instructed contexts. We next consider what might lead to the adoption of such different success criteria in the two contexts. 


\subsection{Representation for selection}

Volition modulation paradigms implicitly assume that alternative actions are represented in the same way even if volitional information processing differs for self-selected and instructed choices. However, the target representation for selection is intrinsically ambiguous in binary choices of the kind investigated here (Haggard 2008). For instance, selection could be objectdirected (i.e., which button to press: the left or right button) or instead be action-directed (i.e., which movement to make: a left or right finger flexion) (Allport 1987; Cisek 2007; Oliveira et al. 2010). Object-directed selection operates on representations of the possible spatial targets for a subsequent action while action-directed selection instead operates on representations of the possible actions. Our narrow vantage point over the motor system provided a test of whether selection might be action-directed in both the Instructed and Free contexts.

The nearly anti-phase interhemispheric coupling ( $\delta$-band) in the Instructed context (Fig. 2,3 ) is consistent with competitive inhibition targeted at the cortical motor representation of the non-selected action in the ipsilateral hemisphere (B. Burle et al. 2004; Borís Burle et al. 2016; Grefkes et al. 2008; Duque et al. 2017). However, the nearly in-phase coupling in the Free context is an unclear indicator of the motor status of the non-selected action. If the postmovement $\beta$-rebound (Fig. 6C,D) is specific to active motor representations then it suggests a resolution. In the Instructed context, the symmetric $\beta$-rebound over both hemispheres is consistent with the motoric representation of both action options. By contrast, the negligible magnitude of the ipsilateral $\beta$-rebound in the Free context implies that only one action (contralateral) was motorically represented in this context but not the other action option (ipsilateral). Taken together, these findings are consistent with selection being action-directed in the Instructed context but not in the Free context. 
We propose that action-directed selection in the Instructed, but not Free, context leads to differing control demands for action execution in the two contexts - specifically a verification that the correct movement was executed when selection is movement-directed, and a verification that there was a successful interaction with the target object when selection is object-directed. These differing control demands consequently lead to the use of a movementrelated criterion (MOV) in the Instructed context and a contact-related criterion $(\mathrm{CON})$ in the Free context. The question of what contextual considerations inform this difference in representations between the Free and Instructed context is an important issue for future studies. The meta-decision about to how to represent the action alternatives in the first place is a major variable in any decision and is informed by broader contextual considerations (Gold and Shadlen 2007; Haggard 2008). One contributing factor to this representation difference could be the self-paced structure of the Free context. Even though the binary choice on each trial of the Free context was free of choice-related performance demands (i.e., accuracy), the timing of the action was subject to performance demands and the risk of errors. The risk of executing an action at an inappropriate time would theoretically be minimal if neither action option was motorically prepared in advance. Object-directed selection might therefore be of strategic relevance to the Free context as the spatial target for the action (i.e., left or right button) could be selected in advance without motorically representing the action until a suitable time is reached. In this way, at the intended time, the action linked to the selected spatial target could be motorically represented and executed in a controlled manner (cf. Haggard and Eimer 1999). Representing only one action would make competitive inhibition of the non-selected action unnecessary in the Free context. 
In conclusion, our findings suggest that, far from being an abstract cognitive activity, volition's effects extend deep into the motor system. 


\section{Acknowledgments}

We thank Svitlana Popovych, Nils Rosjat, Azamat Yeldesbay, Liqing Liu, Eva Niessen, Claudia Schmidt, and Ralph Weidner for invaluable discussions. This work was supported by the University of Cologne Emerging Groups Initiative (CONNECT group) implemented into the Institutional Strategy of the University of Cologne and the German Excellence Initiative. GRF gratefully acknowledges additional support from the Marga and Walter Boll Foundation. SD

gratefully acknowledges support from the German Research Foundation (DA 1935/5-2). The authors declare no competing financial interests. 


\section{Appendix}

Table A.1. ANOVA statistics [Context \{Free, Instructed\} x Movement $\{$ Push,Release $\}$ ] (upper table)(Fig. S2B), and [Context $\{$ Push, Release $\} \times$ Laterality $\{$ Contra,Ipsi $\}]($ lower table)(Fig. S2C). Shaded cells indicate statistically significant effects corrected for multiple comparisons (Bonferroni $\alpha=0.0036$ ) with colors matching those of Figure S2 (B) and (C).

\begin{tabular}{c|cc|cc|cc} 
& \multicolumn{2}{|c|}{ Context*Mvmt } & \multicolumn{2}{|c|}{ Context } & \multicolumn{2}{|c}{ Mvmt } \\
Electrode & $\mathbf{F ( 1 , 1 7 )}$ & $\boldsymbol{p}$ & $\mathbf{F}(\mathbf{1 , 1 7})$ & $\boldsymbol{p}$ & $\mathbf{F}(\mathbf{1 , 1 7})$ & $\boldsymbol{p}$ \\
\hline FC3 & 0.018 & 0.8952 & 0.090 & 0.7684 & 42.917 & 0.0000 \\
FC1 & 1.230 & 0.2828 & 29.216 & 0.0000 & 39.623 & 0.0000 \\
C3 & 2.813 & 0.1118 & 4.690 & 0.0448 & 22.777 & 0.0002 \\
C1 & 0.512 & 0.4838 & 1.197 & 0.2891 & 56.927 & 0.0000 \\
CP5 & 0.079 & 0.7824 & 3.866 & 0.0658 & 3.845 & 0.0665 \\
CP3 & 4.871 & 0.0413 & 37.286 & 0.0000 & 0.634 & 0.4369 \\
CP1 & 0.321 & 0.5785 & 17.145 & 0.0007 & 3.337 & 0.0854
\end{tabular}

\begin{tabular}{c|cc|cc|cc} 
& \multicolumn{2}{|c|}{ Context*Laterality } & \multicolumn{2}{|c|}{ Context } & \multicolumn{2}{|c}{ Laterality } \\
Electrode & $\mathbf{F ( 1 , 1 7 )}$ & $\boldsymbol{p}$ & $\mathbf{F}(\mathbf{1 , 1 7})$ & $\boldsymbol{p}$ & $\mathbf{F}(\mathbf{1 , 1 7})$ & $\boldsymbol{p}$ \\
\hline FC3 & 29.060 & 0.0000 & 3.518 & 0.0780 & 91.407 & 0.0000 \\
FC1 & 35.438 & 0.0000 & 0.036 & 0.8524 & 125.376 & 0.0000 \\
C3 & 10.975 & 0.0041 & 7.954 & 0.0118 & 17.946 & 0.0006 \\
C1 & 21.527 & 0.0002 & 2.994 & 0.1017 & 56.453 & 0.0000 \\
CP5 & 3.479 & 0.0795 & 0.466 & 0.5041 & 40.623 & 0.0000 \\
CP3 & 0.066 & 0.8009 & 45.653 & 0.0000 & 1.278 & 0.2739 \\
CP1 & 3.322 & 0.0860 & 28.334 & 0.0001 & 0.780 & 0.3894
\end{tabular}


Table A.2. Correlations of Push-evoked ERPs to Push and Release latencies for the Instructed context (Fig. 5B) and Free context. Shaded cells indicate statistically significant correlations after correction for multiple comparisons (Bonferroni $\alpha=0.0083$ ).

\begin{tabular}{|c|c|c|c|c|c|c|c|c|}
\hline \multirow{2}{*}{$\begin{array}{c}\text { Push-evoked } \\
\text { ERPs }\end{array}$} & \multicolumn{4}{|c|}{ Instructed context } & \multicolumn{4}{c|}{ Free context } \\
\cline { 2 - 9 } & \multicolumn{2}{|c|}{ Push latency } & \multicolumn{2}{|c|}{ Release latency } & \multicolumn{2}{c|}{ Push latency } & \multicolumn{2}{c|}{ Release latency } \\
\cline { 2 - 9 } & $r(17)$ & $p$ & $r(17)$ & $p$ & $r(17)$ & $p$ & $r(17)$ & $p$ \\
\hline $\mathrm{FC} 1 / 2$ & +0.536 & 0.022 & -0.615 & 0.0066 & +0.379 & 0.121 & -0.389 & 0.110 \\
\hline $\mathrm{CP} 1 / 2$ & +0.654 & 0.0032 & -0.241 & 0.336 & +0.123 & 0.627 & -0.270 & 0.278 \\
\hline $\mathrm{CP} 3 / 4$ & +0.614 & 0.0067 & -0.191 & 0.447 & +0.304 & 0.2193 & -0.198 & 0.431 \\
\hline
\end{tabular}


Table A.3. Full statistics for ANOVA evaluating $\beta$-band amplitude modulation in Figure 6B with factors Context $\{$ Free, Instructed $\}$ x Time $\left\{\mathrm{t}_{1}, . ., \mathrm{t}_{5}\right\}$. Shaded cells indicate statistically significant effects corrected for multiple comparisons (Bonferroni $\alpha=0.0036$ ).

Modulation of Push-evoked $\beta$ amplitude

\begin{tabular}{c|cc|cc|cc} 
& \multicolumn{2}{|c|}{ Context*Time } & \multicolumn{2}{|c|}{ Context } & \multicolumn{2}{|c}{ Time } \\
Electrode & $\mathbf{F ( 4 , 6 8 )}$ & $\boldsymbol{p}$ & $\mathbf{F}(\mathbf{1 , 1 7})$ & $\boldsymbol{p}$ & $\mathbf{F}(\mathbf{4 , 6 8})$ & $\boldsymbol{p}$ \\
\hline FC3 & 2.878 & 0.0290 & 11.746 & 0.0032 & 4.140 & 0.0046 \\
FC1 & 5.657 & 0.0005 & 9.965 & 0.0058 & 3.367 & 0.0142 \\
C3 & 3.820 & 0.0074 & 12.427 & 0.0026 & 4.056 & 0.0052 \\
C1 & 10.275 & 0.0000 & 6.924 & 0.0175 & 8.477 & 0.0000 \\
CP5 & 7.274 & 0.0001 & 16.882 & 0.0007 & 5.288 & 0.0009 \\
CP3 & 9.682 & 0.0000 & 21.404 & 0.0002 & 12.204 & 0.0000 \\
CP1 & 14.103 & 0.0000 & 21.359 & 0.0002 & 15.566 & 0.0000
\end{tabular}

$\underline{\text { Modulation of Release-evoked } \beta \text { amplitude }}$

\begin{tabular}{c|cc|cc|cc} 
& \multicolumn{2}{|c|}{ Context*Time } & \multicolumn{2}{|c|}{ Context } & \multicolumn{2}{|c}{ Time } \\
Electrode & $\mathbf{F ( 4 , 6 8 )}$ & $\boldsymbol{p}$ & $\mathbf{F}(\mathbf{1 , 1 7})$ & $\boldsymbol{p}$ & $\mathbf{F}(\mathbf{4 , 6 8})$ & $\boldsymbol{p}$ \\
\hline $\mathrm{FC} 3$ & 3.728 & 0.0084 & 0.074 & 0.7888 & 4.469 & 0.0029 \\
$\mathrm{FC1}$ & 0.135 & 0.9691 & 0.180 & 0.6766 & 7.928 & 0.0000 \\
$\mathrm{C} 3$ & 2.042 & 0.0982 & 1.684 & 0.2117 & 12.360 & 0.0000 \\
$\mathrm{C} 1$ & 0.834 & 0.5082 & 0.920 & 0.3509 & 13.303 & 0.0000 \\
CP5 & 1.927 & 0.1159 & 0.080 & 0.7801 & 3.888 & 0.0067 \\
CP3 & 3.707 & 0.0087 & 0.210 & 0.6528 & 10.850 & 0.0000 \\
CP1 & 0.513 & 0.7263 & 4.523 & 0.0484 & 13.005 & 0.0000
\end{tabular}




\section{REFERENCES}

Alegre, Manuel, I. G. De Gurtubay, Alberto Labarga, Jorge Iriarte, Armando Malanda, and Julio Artieda. 2004. "Alpha and Beta Oscillatory Activity during a Sequence of Two Movements." Clinical Neurophysiology 115 (1): 124-30. https://doi.org/10.1016/S13882457(03)00311-0.

Allport, A. 1987. "Selection for Action: Some Behavioral and Neurophysiological Considerations of Attention and Action." In Perspectives on Perception and Action, 395419.

Ariani, G., M. F. Wurm, and a. Lingnau. 2015. "Decoding Internally and Externally Driven Movement Plans.” Journal of Neuroscience 35 (42): 14160-71. https://doi.org/10.1523/JNEUROSCI.0596-15.2015.

Aschersleben, Gisa. 2002. "Temporal Control of Movements in Sensorimotor Synchronization." Brain and Cognition 48 (1): 66-79. https://doi.org/10.1006/brcg.2001.1304.

Aschersleben, Gisa, Jörg Gehrke, and Wolfgang Prinz. 2001. "Tapping with Peripheral Nerve Block.” Experimental Brain Research 136 (3): 331-39. https://doi.org/10.1007/s002210000562.

Becchio, Cristina, Debora Zanatto, Elisa Straulino, Andrea Cavallo, Giuseppe Sartori, and Umberto Castiello. 2014. "The Kinematic Signature of Voluntary Actions." Neuropsychologia 64. Elsevier: 169-75. https://doi.org/10.1016/j.neuropsychologia.2014.09.033.

Berens, Philipp. 2009. “CircStat: A MATLAB Toolbox for Circular Statistics.” Journal of Statistical Software 31 (10): 1-21. https://doi.org/10.1002/wics.10. 
Bode, Stefan, Carsten Bogler, and John Dylan Haynes. 2013. "Similar Neural Mechanisms for Perceptual Guesses and Free Decisions.” NeuroImage 65. Elsevier Inc.: 456-65. https://doi.org/10.1016/j.neuroimage.2012.09.064.

Bode, Stefan, Carsten Murawski, Chun Siong Soon, Philipp Bode, Jutta Stahl, and Philip L. Smith. 2014. 'Demystifying 'Free Will': The Role of Contextual Information and Evidence Accumulation for Predictive Brain Activity." Neuroscience and Biobehavioral Reviews 47. Elsevier Ltd: 636-45. https://doi.org/10.1016/j.neubiorev.2014.10.017.

Botvinick, Matthew M., Jonathan D. Cohen, and Cameron S. Carter. 2004. “Conflict Monitoring and Anterior Cingulate Cortex: An Update.” Trends in Cognitive Sciences 8 (12): 539-46. https://doi.org/10.1016/j.tics.2004.10.003.

Brass, Marcel, and Patrick Haggard. 2008. “The What, When, Whether Model of Intentional Action." The Neuroscientist : A Review Journal Bringing Neurobiology, Neurology and Psychiatry 14 (4): 319-25. https://doi.org/10.1177/1073858408317417.

Brass, Marcel, Margaret T. Lynn, Jelle Demanet, and Davide Rigoni. 2013. 'Imaging Volition: What the Brain Can Tell Us about the Will.” Experimental Brain Research 229 (3): 30112. https://doi.org/10.1007/s00221-013-3472-X.

Burle, B., F. Vidal, C Tandonnet, and T Hasbroucq. 2004. "Physiological Evidence for Response Inhibition in Choice Reaction Time Tasks.” Brain and Cognition 56 (2): 153-64. https://doi.org/10.1016/j.bandc.2004.06.004.

Burle, Borís, Wery P M van den Wildenberg, Laure Spieser, and K. Richard Ridderinkhof. 2016. "Preventing (Impulsive) Errors: Electrophysiological Evidence for Online Inhibitory Control over Incorrect Responses.” Psychophysiology 53 (7): 1008-19. 
https://doi.org/10.1111/psyp.12647.

Cassim, François, William Szurhaj, Haouaria Sediri, David Devos, Jean Louis Bourriez, Isabelle Poirot, Philippe Derambure, Luc Defebvre, and Jean Daniel Guieu. 2000. "Brief and Sustained Movements: Differences in Event-Related (de)Synchronization (ERD/ERS) Patterns." Clinical Neurophysiology 111 (11): 2032-39. https://doi.org/10.1016/S13882457(00)00455-7.

Cheyne, Douglas Owen. 2013. "MEG Studies of Sensorimotor Rhythms: A Review." Experimental Neurology 245. Elsevier B.V.: 27-39. https://doi.org/10.1016/j.expneurol.2012.08.030.

Cisek, Paul. 2007. "Cortical Mechanisms of Action Selection: The Affordance Competition Hypothesis." Philosophical Transactions of the Royal Society of London. Series B, Biological Sciences 362 (1485): 1585-99. https://doi.org/10.1098/rstb.2007.2054.

Cohen, Mike X. 2014. “Analyzing Neural Time Series Data: Theory and Practice.” MIT Press, 600. https://doi.org/10.1017/CBO9781107415324.004.

Cousineau, Denis. 2005. “Confidence Intervals in Within-Subject Designs: A Simpler Solution to Loftus and Masson's Method." Tutorials in Quantitative Methods for Psychology 1 (1): 42-45. https://doi.org/no DOI found.

Cunnington, R, C Windischberger, L Deecke, and E Moser. 2002. "The Preparation and Execution of Self-Initiated and Externally-Triggered Movement: A Study of Event-Related FMRI." NeuroImage 15 (2): 373-85. https://doi.org/10.1006/nimg.2001.0976.

Delorme, Arnaud, and Scott Makeig. 2004. "EEGLAB: An Open Source Toolbox for Analysis of Single-Trial EEG Dynamics Including Independent Component Analysis." Journal of 
Neuroscience Methods 134 (1): 9-21. https://doi.org/10.1016/j.jneumeth.2003.10.009.

Desmurget, M, and S Grafton. 2000. "Forward Modeling Allows Feedback Control for Fast Reaching Movements.” Trends in Cognitive Sciences 4 (11): 423-31. http://www.ncbi.nlm.nih.gov/pubmed/11058820.

Desmurget, Michel, Karen T Reilly, Nathalie Richard, Alexandru Szathmari, Carmine Mottolese, and Angela Sirigu. 2009. "Movement Intention after Parietal Cortex Stimulation in Humans." Science (New York, N.Y.) 324 (5928): 811-13. https://doi.org/10.1126/science.1169896.

Desmurget, Michel, and Angela Sirigu. 2009. “A Parietal-Premotor Network for Movement Intention and Motor Awareness." Trends in Cognitive Sciences 13 (10): 411-19. https://doi.org/10.1016/j.tics.2009.08.001.

Duque, Julie, Ian Greenhouse, Ludovica Labruna, and Richard B. Ivry. 2017. "Physiological Markers of Motor Inhibition during Human Behavior." Trends in Neurosciences 40 (4). Elsevier Ltd: 219-36. https://doi.org/10.1016/j.tins.2017.02.006.

Eimeren, Thilo Van, Thomas Wolbers, Alexander Münchau, Christian Büchel, Cornelius Weiller, and Hartwig Roman Siebner. 2006. "Implementation of Visuospatial Cues in Response Selection." NeuroImage 29 (1): 286-94. https://doi.org/10.1016/j.neuroimage.2005.07.014.

Erbil, Nurhan, and Pekcan Ungan. 2007. "Changes in the Alpha and Beta Amplitudes of the Central EEG during the Onset, Continuation, and Offset of Long-Duration Repetitive Hand Movements." Brain Research 1169 (2004): 44-56. https://doi.org/10.1016/j.brainres.2007.07.014. 
Freeman, Walter J. 2004. “Origin, Structure, and Role of Background EEG Activity. Part 1. Analytic Amplitude." Clinical Neurophysiology 115 (9): 2077-88. https://doi.org/10.1016/j.clinph.2004.02.029.

Frith, C. D., K. Friston, P. F. Liddle, and R. S. J. Frackowiak. 1991. "Willed Action and the Prefrontal Cortex in Man: A Study with PET." Proceedings of the Royal Society B:

Biological Sciences 244 (1311): 241-46. https://doi.org/10.1098/rspb.1991.0077.

Goebl, Werner, and Caroline Palmer. 2008. "Tactile Feedback and Timing Accuracy in Piano Performance," 471-79. https://doi.org/10.1007/s00221-007-1252-1.

Gold, Joshua I., and Michael N. Shadlen. 2007. "The Neural Basis of Decision Making.” Annual Review of Neuroscience 30 (1): 535-74. https://doi.org/10.1146/annurev.neuro.29.051605.113038.

Gordon, Andrew M, and John F Soeehting. 1995. "Use of Tactile Afferent Information in Sequential Finger Movements," 281-92.

Grefkes, Christian, Simon B Eickhoff, Dennis A Nowak, Manuel Dafotakis, and Gereon R Fink. 2008. "Dynamic Intra- and Interhemispheric Interactions during Unilateral and Bilateral Hand Movements Assessed with FMRI and DCM.” NeuroImage 41 (4): 1382-94. https://doi.org/10.1016/j.neuroimage.2008.03.048.

Grondin, Simon. 2010. “Timing and Time Perception: A Review of Recent Behavioral and Neuroscience Findings and Theoretical Directions.” Attention, Perception, \& Psychophysics 72 (3): 561-82. https://doi.org/10.3758/APP.72.3.561.

Haggard, Patrick. 2008. "Human Volition: Towards a Neuroscience of Will.” Nature Reviews. Neuroscience 9 (12): 934-46. https://doi.org/10.1038/nrn2497. 
Haggard, Patrick, and Martin Eimer. 1999. "On the Relation between Brain Potentials and the Awareness of Voluntary Movements.” Experimental Brain Research 126 (1): 128-33. https://doi.org/10.1007/s002210050722.

Hamel-Thibault, Audrey, François Thénault, Kevin Whittingstall, and Pierre-Michel Bernier. 2016. "Delta-Band Oscillations in Motor Regions Predict Hand Selection for Reaching." Cerebral Cortex (New York, N.Y. : 1991), 1-11. https://doi.org/10.1093/cercor/bhw392.

Hoffstaedter, Felix, Christian Grefkes, Svenja Caspers, Christian Roski, Nicola PalomeroGallagher, Angie R. Laird, Peter T. Fox, and Simon B. Eickhoff. 2014. "The Role of Anterior Midcingulate Cortex in Cognitive Motor Control: Evidence from Functional Connectivity Analyses.” Human Brain Mapping 35 (6): 2741-53. https://doi.org/10.1002/hbm.22363.

Hoffstaedter, Felix, Christian Grefkes, Karl Zilles, and Simon B. Eickhoff. 2013. "The 'What' and 'When' of Self-Initiated Movements.' Cerebral Cortex 23 (3): 520-30. https://doi.org/10.1093/cercor/bhr391.

Hughes, G., S. Schutz-Bosbach, and F. Waszak. 2011. “One Action System or Two? Evidence for Common Central Preparatory Mechanisms in Voluntary and Stimulus-Driven Actions.” Journal of Neuroscience 31 (46): 16692-99. https://doi.org/10.1523/JNEUROSCI.225611.2011.

Jahanshahi, M, I H Jenkins, R G Brown, C D Marsden, R E Passingham, and D J Brooks. 1995. “Self-Initiated Versus Externally Triggered Movements .1. an Investigation Using Measurement of Regional Cerebral Blood-Flow With Pet and Movement-Related Potentials in Normal and Parkinsons-Disease Subjects.” Brain 118: 913-33. 
https://doi.org/10.1093/brain/119.3.1045.

Jenkins, I H, M Jahanshahi, M Jueptner, R E Passingham, and D J Brooks. 2000. "Self-Initiated versus Externally Triggered Movements. II. The Effect of Movement Predictability on Regional Cerebral Blood Flow.” Brain : A Journal of Neurology 123 ( Pt 6: 1216-28. https://doi.org/10.1093/brain/123.6.1216.

Jurkiewicz, Michael T., William C. Gaetz, Andreea C. Bostan, and Douglas Cheyne. 2006. "Post-Movement Beta Rebound Is Generated in Motor Cortex: Evidence from Neuromagnetic Recordings.” NeuroImage 32 (3): 1281-89. https://doi.org/10.1016/j.neuroimage.2006.06.005.

Kayser, Jürgen, and Craig E. Tenke. 2006. "Principal Components Analysis of Laplacian Waveforms as a Generic Method for Identifying ERP Generator Patterns: II. Adequacy of Low-Density Estimates.” Clinical Neurophysiology 117 (2): 369-80. https://doi.org/10.1016/j.clinph.2005.08.033.

Kilavik, Bjørg Elisabeth, Manuel Zaepffel, Andrea Brovelli, William A. MacKay, and Alexa Riehle. 2013. "The Ups and Downs of Beta Oscillations in Sensorimotor Cortex." Experimental Neurology 245. Elsevier Inc.: 15-26. https://doi.org/10.1016/j.expneurol.2012.09.014.

Kornhuber, HH, and L Deecke. 1964. "Hirnpotentialänderungen Beim Menschen Vor Und Nach Willkurbewegungen, Dargestellt Mit Magnetband-Speiche- Rung Und Ruckwartsanalyse.” Pflugers Arch 281: 52.

Krieghoff, Veronika, Florian Waszak, Wolfgang Prinz, and Marcel Brass. 2011. "Neural and Behavioral Correlates of Intentional Actions.” Neuropsychologia 49 (5). Elsevier Ltd: $767-$ 
76. https://doi.org/10.1016/j.neuropsychologia.2011.01.025.

Kutas, Marta, and Emanuel Donchin. 1980. "Preparation to Response as Manifested by Movement-Related Brain Potentials.” Brain Research 202: 95-115.

Lau, H. C., R. D. Rogers, Patrick Haggard, and R. E. Passingham. 2004. "Attention to Intention." Science 303 (5661): 1208-10. https://doi.org/10.1126/science.1090973.

Libet, B., C. A. Gleason, E. W. Wright, and D. K. Pearl. 1983. “Time of Conscious Intention To Act in Relation To Onset of Cerebral Activity (Readiness-Potential)." Brain 106 (3): 623 42. https://doi.org/10.1093/brain/106.3.623.

Libet, B., E. W. Wright, and C. A. Gleason. 1982. "Readiness-Potentials Preceding Unrestricted ‘spontaneous' vs. Pre-Planned Voluntary Acts.” Electroencephalography and Clinical Neurophysiology 54 (3): 322-35. https://doi.org/10.1016/0013-4694(82)90181-X.

Miall, R. C., and D. M. Wolpert. 1996. "Forward Models for Physiological Motor Control.” Neural Networks. https://doi.org/10.1016/S0893-6080(96)00035-4.

Michely, Jochen, Michael T. Barbe, Felix Hoffstaedter, Lars Timmermann, Simon B. Eickhoff, Gereon R. Fink, and Christian Grefkes. 2012. "Differential Effects of Dopaminergic Medication on Basic Motor Performance and Executive Functions in Parkinson's Disease.” Neuropsychologia 50 (10). Elsevier: 2506-14. https://doi.org/10.1016/j.neuropsychologia.2012.06.023.

Michely, Jochen, L. J. Volz, M. T. Barbe, F. Hoffstaedter, S. Viswanathan, L. Timmermann, S. B. Eickhoff, G. R. Fink, and C. Grefkes. 2015. "Dopaminergic Modulation of Motor Network Dynamics in Parkinson's Disease.” Brain 138 (3): 664-78. https://doi.org/10.1093/brain/awu381. 
Mognon, Andrea, Jorge Jovicich, Lorenzo Bruzzone, and Marco Buiatti. 2011. “ADJUST: An Automatic EEG Artifact Detector Based on the Joint Use of Spatial and Temporal Features." Psychophysiology 48 (2): 229-40. https://doi.org/10.1111/j.14698986.2010.01061.x.

Mueller, Veronika A., Marcel Brass, Florian Waszak, and Wolfgang Prinz. 2007. "The Role of the PreSMA and the Rostral Cingulate Zone in Internally Selected Actions." NeuroImage 37 (4): 1354-61. https://doi.org/10.1016/j.neuroimage.2007.06.018.

Nachev, Parashkev, and Masud Husain. 2010. "Action and the Fallacy of the 'Internal': Comment on Passingham et Al.” Trends in Cognitive Sciences 14 (5): 192-93. https://doi.org/10.1016/j.tics.2010.02.006.

Nachev, Parashkev, Geraint Rees, Andrew Parton, Christopher Kennard, and Masud Husain. 2005. "Volition and Conflict in Human Medial Frontal Cortex." Current Biology 15 (2): 122-28. https://doi.org/10.1016/j.cub.2005.01.006.

Nunez, P.L., R.B. Silberstein, P.J. Cadusch, R.S. Wijesinghe, a.F. Westdorp, and R. Srinivasan. 1994. "A Theoretical and Experimental Study of High Resolution EEG Based on Surface Laplacians and Cortical Imaging." Electroencephalography and Clinical Neurophysiology 90 (1): 40-57. https://doi.org/10.1016/0013-4694(94)90112-0.

O'Connell, Redmond G., Michael N. Shadlen, KongFatt Wong-Lin, and Simon P. Kelly. 2018. "Bridging Neural and Computational Viewpoints on Perceptual Decision Making (Accepted)." Trends in Neurosciences xx. Elsevier Ltd: 1-15. https://doi.org/10.1016/j.tins.2018.06.005.

Obhi, Sukhvinder S., and Patrick Haggard. 2004. "Internally Generated and Externally 
Triggered Actions Are Physically Distinct and Independently Controlled.” Experimental Brain Research 156 (4): 518-23. https://doi.org/10.1007/s00221-004-1911-4.

Obhi, Sukhvinder S., Shannon Matkovich, and Sam J. Gilbert. 2009. "Modification of Planned Actions." Experimental Brain Research 192 (2): 265-74. https://doi.org/10.1007/s00221008-1584-5.

Oldfield, R.C. 1971. "The Assessment and Analysis of Handedness: The Edinburgh Inventory." Neuropsychologia 9 (1): 97-113. https://doi.org/10.1016/0028-3932(71)90067-4.

Oliveira, Flavio T P, Jörn Diedrichsen, Timothy Verstynen, Julie Duque, and Richard B Ivry. 2010. "Transcranial Magnetic Stimulation of Posterior Parietal Cortex Affects Decisions of Hand Choice." Proceedings of the National Academy of Sciences of the United States of America 107 (41): 17751-56. https://doi.org/10.1073/pnas.1006223107.

Oulasvirta, Antti, Sunjun Kim, and Byungjoo Lee. 2018. "Neuromechanics of a Button Press." In Proceedings of the 2018 CHI Conference on Human Factors in Computing Systems (CHI '18), 4099-4112. ACM Press. https://doi.org/10.1145/3173574.3174082.

Passingham, Richard E., Sara L. Bengtsson, and Hakwan C. Lau. 2010. "Medial Frontal Cortex: From Self-Generated Action to Reflection on One's Own Performance.” Trends in Cognitive Sciences 14 (1). Elsevier Ltd: 16-21. https://doi.org/10.1016/j.tics.2009.11.001.

Perrin, F, J Pernier, O Bertrand, and J F Echallier. 1989. "Spherical Splines for Scalp Potential and Current Density Mapping." Electroencephalography and Clinical Neurophysiology 72 (2): 184-87. https://doi.org/10.1016/0013-4694(89)90180-6.

Pfurtscheller, G, and F H Lopes da Silva. 1999. "Event-Related EEG/MEG Synchronization and Desynchronization: Basic Principles." Clinical Neurophysiology: Official Journal of the 
International Federation of Clinical Neurophysiology 110 (11): 1842-57.

http://www.ncbi.nlm.nih.gov/pubmed/10576479.

Popovych, S, N Rosjat, T.I. Toth, B.A. Wang, L Liu, R.O. Abdollahi, S Viswanathan, C Grefkes, G.R. Fink, and S Daun. 2016. "Movement-Related Phase Locking in the DeltaTheta Frequency Band.” NeuroImage 139 (October). Elsevier Inc.: 439-49. https://doi.org/10.1016/j.neuroimage.2016.06.052.

Rabin, Ely, and Andrew M. Gordon. 2004. "Tactile Feedback Contributes to Consistency of Finger Movements during Typing.” Experimental Brain Research 155 (3): 362-69. https://doi.org/10.1007/s00221-003-1736-6.

Ratcliff, R. 1993. "Methods for Dealing with Reaction Time Outliers." Psychological Bulletin 114 (3): 510-32. https://doi.org/10.1037/0033-2909.114.3.510.

Ratcliff, Roger. 2008. “The Diffusion Decision Model : Theory and Data for Two-Choice Decision Tasks" 922: 873-922.

Roskies, Adina L. 2010. "How Does Neuroscience Affect Our Conception of Volition?” Annual Review of Neuroscience 33 (1): 109-30. https://doi.org/10.1146/annurev-neuro-060909153151.

Schouten, J F, and J a M Bekker. 1967. "Reactlon Tine and Accuracy." Acta Psychologica 27: $143-53$.

Schurger, Aaron, J. D. Sitt, and S. Dehaene. 2012. “An Accumulator Model for Spontaneous Neural Activity Prior to Self-Initiated Movement." Proceedings of the National Academy of Sciences 109 (42): E2904-13. https://doi.org/10.1073/pnas.1210467109.

Schurger, Aaron, and Sebo Uithol. 2015. "Nowhere and Everywhere: The Causal Origin of 
Voluntary Action.” Review of Philosophy and Psychology 6 (4): 761-78.

https://doi.org/10.1007/s13164-014-0223-2.

Schüür, Friederike, and Patrick Haggard. 2011. "What Are Self-Generated Actions?"

Consciousness and Cognition 20 (4): 1697-1704.

https://doi.org/10.1016/j.concog.2011.09.006.

Selen, Luc P J, Michael N Shadlen, and Daniel M Wolpert. 2012. "Deliberation in the Motor System: Reflex Gains Track Evolving Evidence Leading to a Decision." The Journal of Neuroscience: The Official Journal of the Society for Neuroscience 32 (7): 2276-86. https://doi.org/10.1523/JNEUROSCI.5273-11.2012.

Shibasaki, Hiroshi, and Mark Hallett. 2006. "What Is the Bereitschaftspotential?" Clinical Neurophysiology : Official Journal of the International Federation of Clinical Neurophysiology 117 (11): 2341-56. https://doi.org/10.1016/j.clinph.2006.04.025.

Soon, Chun Siong, Marcel Brass, Hans-Jochen Heinze, and John-Dylan Haynes. 2008. “Unconscious Determinants of Free Decisions in the Human Brain.” Nature Neuroscience 11 (5): 543-45. https://doi.org/10.1038/nn.2112.

Thura, David, and Paul Cisek. 2014. "Deliberation and Commitment in the Premotor and Primary Motor Cortex during Dynamic Decision Making." Neuron 81 (6). Elsevier Inc.: 1401-16. https://doi.org/10.1016/j.neuron.2014.01.031.

Wang, Bin A, Shivakumar Viswanathan, Rouhollah O Abdollahi, Nils Rosjat, Svitlana Popovych, Silvia Daun, Christian Grefkes, and Gereon R Fink. 2017. “Frequency-Specific Modulation of Connectivity in the Ipsilateral Sensorimotor Cortex by Different Forms of Movement Initiation.” NeuroImage 159 (October). Elsevier Inc.: 248-60. 
https://doi.org/10.1016/j.neuroimage.2017.07.054.

Waszak, Florian, Edmund Wascher, Peter Keller, Iring Koch, Gisa Aschersleben, David a.

Rosenbaum, and Wolfgang Prinz. 2005. "Intention-Based and Stimulus-Based Mechanisms in Action Selection." Experimental Brain Research 162 (3): 346-56. https://doi.org/10.1007/s00221-004-2183-8.

Wegner, Daniel M. 2002. The Illusion of Conscious Will. MIT Press, Cambridge, MA.

Wisniewski, David, Thomas Goschke, and John-dylan Haynes. 2016. "Similar Coding of Freely Chosen and Externally Cued Intentions in a Fronto-Parietal Network.” NeuroImage 134. Elsevier B.V.: 450-58. https://doi.org/10.1016/j.neuroimage.2016.04.044.

Zapparoli, Laura, Silvia Seghezzi, and Eraldo Paulesu. 2017. "The What, the When and the Whether of Intentional Action in the Brain: A Meta-Analytical Review.” Front. Hum. Neurosci. 11 (May): 1-11. https://doi.org/10.3389/fnhum.2017.00238. 


\section{Supplementary Information}

\section{Freely chosen and instructed actions are terminated by}

\section{different neural mechanisms revealed by kinematics-informed \\ EEG}

Shivakumar Viswanathan, Bin A. Wang, Rouhollah O. Abdollahi, Silvia Daun, Christian Grefkes, Gereon R. Fink 


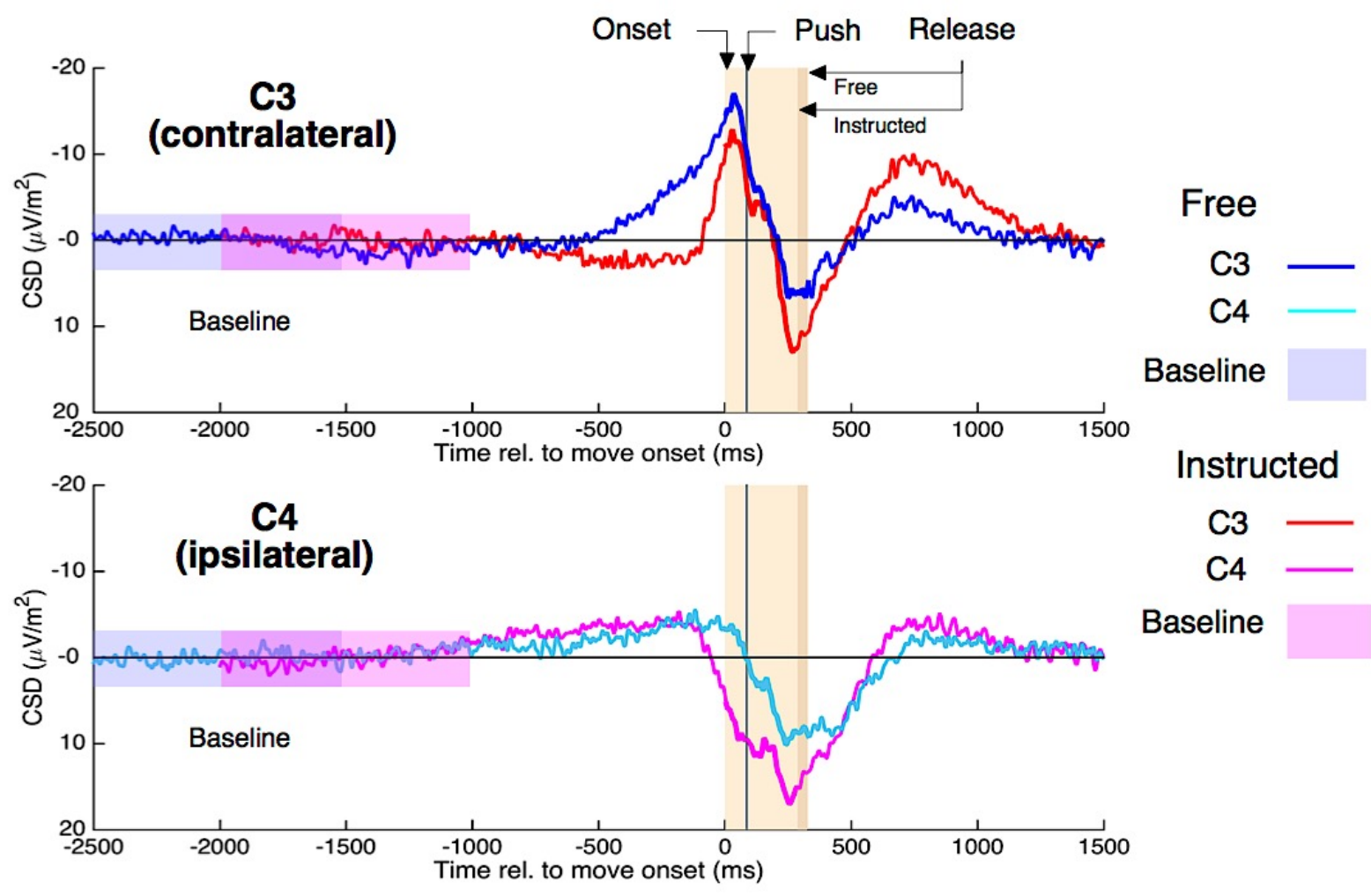

Fig. S1. ERPs at C3 and C4 in the Free and Instructed contexts. The contralateral ERPs at C3 (upper panel) were similar in both contexts at and following movement onset, but the ipsilateral ERPs at C4 (lower panels) were considerably different. The baseline period was $1000 \mathrm{~ms}$ long for both contexts but the pre-movement duration of the epochs was different $([-2500 \mathrm{~ms}, 0 \mathrm{~ms}]$ in the Free context and [-2000ms,0ms] in the Instructed context) to accommodate differences in how responses were selected (see Materials and Methods). 


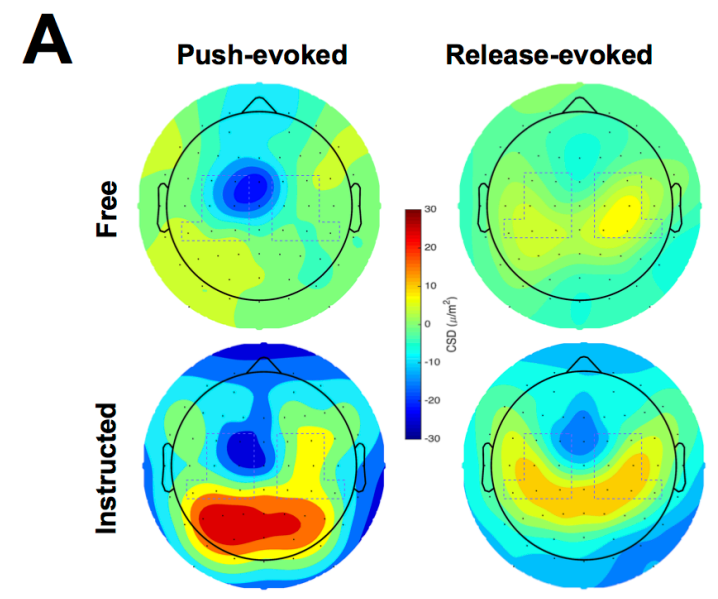

B

Movement-evoked ERP: Context x Mvmt
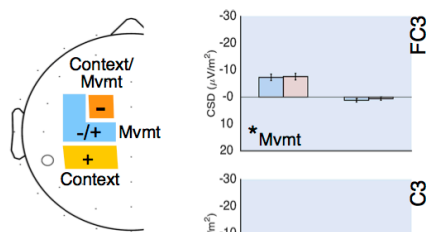

(10.0.
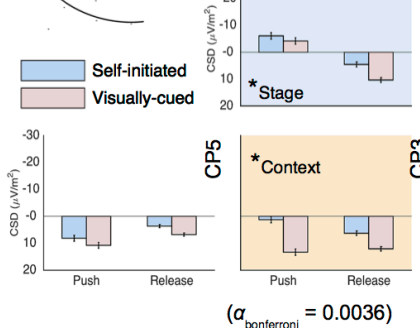

C
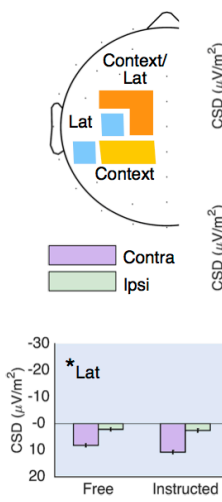
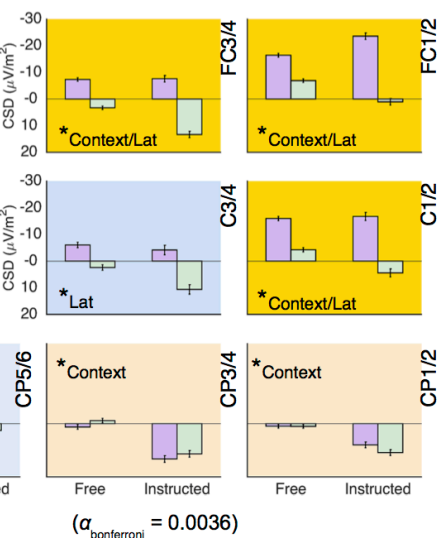

Fig. S2. Push-evoked and Release-evoked ERPs. (A) Topographic distribution of Pushevoked and Release-evoked ERPs. (B) The layout of the bar plots corresponds to the location of electrodes on the scalp. The background color indicates the factor that was statistically significant (Table A.1), and corresponds to the colors on topographic inset. ERPs at FC3/C3/C1 (background color blue) only had a significant effect of movement but not context with ERPs having both positive and negative polarities (+/-). CP1 and CP3 had ERPs with a positive polarity $(+)$ and showed an effect of context but not movement. FC1 showed effects of context and movement. Push-evoked ERPs at FC1, CP1 and CP3 were significantly different between contexts (C) Spatial gradient of Context x Laterality effects (Table A.1) shows that context-dependent lateralization was primarily a property of central and frontocentral electrodes. 


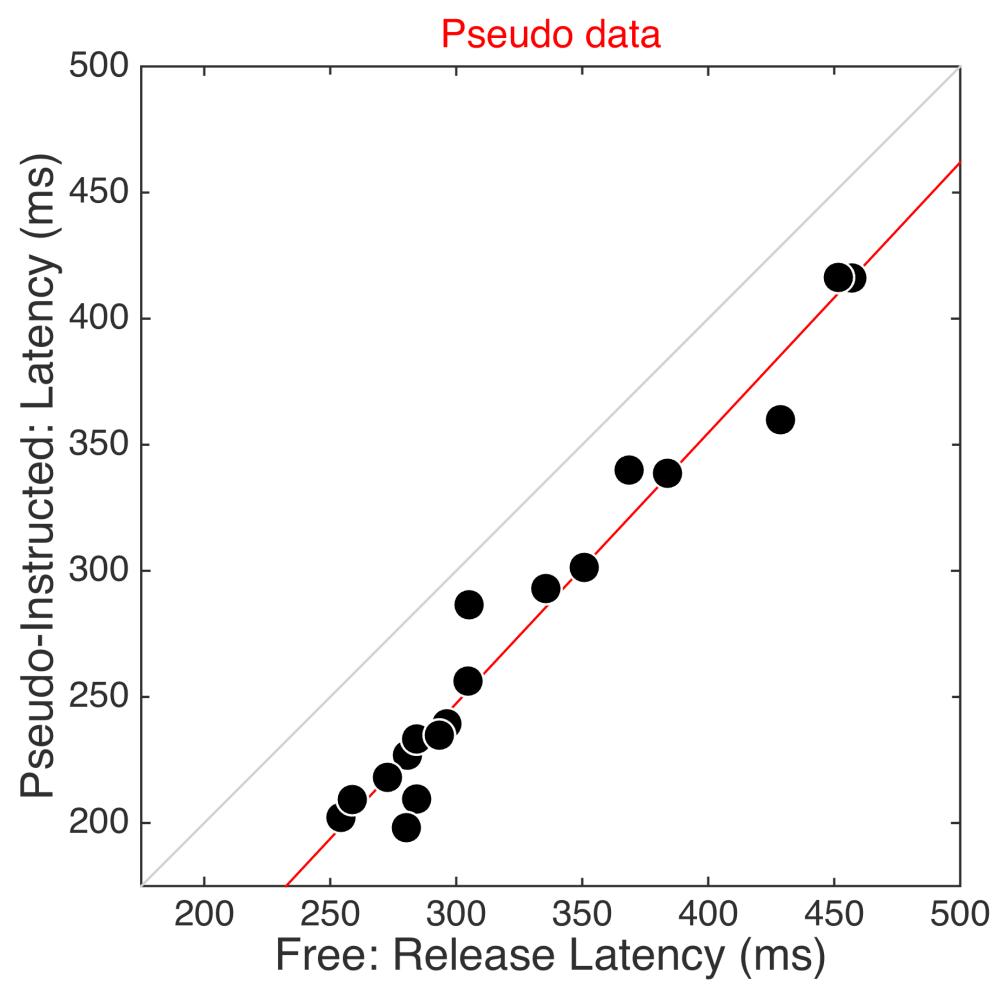

Fig. S3. Example of an additive relationship. A hypothetical linear relationship between Release latency in the Free and Instructed contexts with a mean difference of $50 \mathrm{~ms}$ between contexts that was additive $\left(L_{\mathrm{Instr}} \approx L_{\mathrm{Free}}+\mathrm{Z}\right)$. Pseudo-Release latencies in Instructed context were generated from the true values in the Free context by adding a random value drawn from normal distribution with mean $50 \mathrm{~ms}$ and standard deviation of $20 \mathrm{~ms}$. Consistent with an additive relationship, the best-fit line was parallel to the unit slope line. 